\title{
FURTHER DESCRIPTION AND ANALYSIS OF THE FIRST SPECTRUM OF XENON
}

\author{
By C. J. Humphreys and W. F. Meggers
}

\section{ABSTRACT}

An entirely new description of the first spectrum of Xenon has been completed. Geissler tubes with a small bore capillary, exposed end on, were used as sources and afforded sufficient intensity to permit the use of spectrographs of high resolving power to a greater extent than in the preliminary investigation previously reported. The new description covers the region between 3,340 and 11,140 A. The spectrum has been photographed by aid of a Rowland 20,000-line per inch grating over the range included between 3,440 and 9,800 A. Interferometer measurements have been made on 130 lines, including 45 reported in a previous paper. Several new lines have been found in the ultra-violet between 3,340 and 3,440 where the use of a source of higher intensity has led to the detection of several higher members of the $1 s_{5}-m p_{i}$ series. Most of the new data have been obtained in the infra-red region. Each of the new types of Eastman red and infra-red sensitive plates, $F, N, P$, and $Q$ have been exposed to the radiation in the spectral region for which their respective sensitivity is greatest. It is believed that the spectrum has been completely recorded throughout the region investigated.

Of the 538 lines included in the description all but 13 have been classified. Almost all terms predicted by the Hund theory have been obtained. The new term table embodies a small change in the absolute term values indicated by new measurements of greater precision, several newly discovered terms, and extensions to practically all the series. Some rearrangement of the $d$-terms has been made necessary, due to the elimination of uncertainties in the assignment of $j$-values. A new $f$-type sequence, lying very close to $m Z$, and designated by $m T$, has been found.

\section{CONTENTS}

I. Introduction

II. Experimental details

III. Wave-length measurements.

IV. Discussion of $\mathrm{Xe} I$ terms.

\section{INTRODUCTION}

A preliminary description and analysis of the first spectrum of xenon was published in 1929. ${ }^{1}$ As in the case of the investigation of $\mathrm{Kr}$ I which was undertaken simultaneously, it was expected to give in later publications extensions of the series to higher members permitted by the detection of fainter lines, interferometer comparisons of wave lengths, and measurements of hyperfine structures. Interferometer measurements of 45 xenon lines, ${ }^{2}$ and measurements of the hyperfine structures of 6 xenon lines, ${ }^{3}$ are presented in two papers by

1 W. F. Meggers, T. L. deBruin, and C. J. Humphreys, B. S. Jour. Research, vol. 3 (RP115), p. 731, 1929.

2 C. J. Humphreys, B. S. Jour. Research, vol. 5 (RP245), p. $1041,1930$.

3 C. J. Humphreys, B. S. Jour. Research, vol. 7 (R P351), p. 453, 1931. 
one of us in which the results of a similar investigation of $\mathrm{Kr}$ I are given. In the case of the first spectrum of krypton it was found that a large number of new lines could be recorded by using as a source a Geissler tube exposed end on. A much more intense illumination of the slit could be obtained in this manner than with the usual type of tube, the construction of which permits only side-on exposures. Extended and improved measurements of $\mathrm{Kr}$ I are contained in a paper published about a year ago. ${ }^{4}$ The present paper represents a more complete description of $\mathrm{Xe} I$ than could then be made in the case of krypton because in the meantime new photosenstitizing dyes have been discovered which have greatly extended both the speed of recording and the upper limit of the range of investigation in the infra-red region. All the rare gas spectra have been reinvestigated, using these specially sensitized plates, and "end on" Geissler tubes (from Robert Goetze in Leipzig). The results for those other than xenon are being published in separate papers. The use of more intense sources and more sensitive plates has made possible additional interferometer comparisons, the results of which for Xe I lines are incorporated in the present description. The application of these superior infra-red sensitive photographic plates to spectrographic investigations has created a demand for accurate standards of wave length in the extended range now accessible and it appears that the emission spectra of the noble gases will be useful for this purpose. The results for Xe I lines presented here may be regarded as a contribution to standard wave lengths, since many of the lines have been measured interferometrically either in terms of neon standards or of sharp xenon lines so determined and enough of the spectral terms have thus been accurately fixed in relative value to guarantee the calculated values of the remaining classified lines to within $0.01 \mathrm{~A}$.

Since the publication of our first paper, the spectrum of $\mathrm{X}$ I h has been investigated by Gremmer ${ }^{5}$ and by Rasmussen. ${ }^{6}$ Gremmer's classification is essentially the same as ours. He succeeded in obtaining 16 classified lines not recorded in our first description in the ultraviolet region. These are in all cases higher members of known series. Rasmussen found a considerable number of lines in the infra-red, gave classifications for 80 additional lines, and introduced a number of changes in term designations which are confirmed by the present investigation.

\section{EXPERIMENTAL DETAILS}

New spectograms have been made over the entire region of the Xe I spectrum accessible to photography in air. The Hilger E1 spectograph was used to describe the ultra-violet region. The last recorded line appears at $3,340 \mathrm{~A}$. There is no Xe I radiation to be expected between the $1 s_{5}$ series limit at $3,250 \mathrm{~A}$ and the Schumann region. Between 3,440 and 9,800 A the Rowland grating with 20,000 lines per inch was used, which gives a scale of $3.7 \mathrm{~A} / \mathrm{mm}$ in the first order spectrum. The description previously published (RP115) was made for the most part with a grating of 7,500 lines per inch, giving a scale of $10.4 \mathrm{~A} / \mathrm{mm}$. The region between 6,600 and $8,600 \mathrm{~A}$ was also photographed with a scale of $2.7 \mathrm{~A} / \mathrm{mm}$, given by a plane grating with 20,000 lines per inch and lenses of 16 feet focal length. The published obser-

iW. F. Meggers, T. L. de Bruin, C. J. Humphreys, B. S. Jour. Research, vol. 7 (RP 364), p. 643, 1931.

W. Gremmer, Zeit. $t$. Physik, vol. 59 , p. 154, 1930.

- E. Rasmussen, Zeit. .. I'hysik, vol. 73, p. 779, 1932. 
vations beyond 9,800 A have been made with the 7,500-line-per-inch grating, although the infra-red region was also photographed by aid of a Hilger E2 prism spectograph with gllass optics. Interferometer measurements have been made of the wave lengths of 130 Xe lines including those reported in RP245. For lines shown by the interference observations to have hyperfine structure, the wave length of the strongest component only is here reported. A pair of crystal quartz interferometer plates of $60 \mathrm{~mm}$ aperture, silvered by the evaporation method, have been used in the more recent work. The fixed étalon method has been employed, using invar separators of $3,6,10$, 15 , and $25 \mathrm{~mm}$ length.

In the red and infra-red regions, plates prepared with the new types of sensitizers developed by the Eastman laboratories and designated F, N, P, and Q have been used. The characteristics of these special plates are discussed in a recent paper by Mees. ${ }^{7}$ We have used the $\mathrm{F}$ plate to cover the region from 5,000 to $6,600 \mathrm{~A}$, the $\mathrm{N}$ plate from 6,500 to 8,500 , the P plate between 8,000 and 9,000 , and the Q plate above 9,000 . There is considerable overlapping of the regions of sensitivity of these emulsions except that the F type falls off rather abruptly above $6,800 \mathrm{~A}$. The $\mathrm{N}$ plate shows two maxima near 7,000 and $8,200 \mathrm{~A}$. The respective maxima of the $\mathrm{P}$ and $\mathrm{Q}$ types lie at 8,600 and 9,700 A, respectively. Exposures of from 2 to 24 hours have been made. One exposure was prolonged to 40 hours in order to observe the resolution of the pair of lines at 9,374A with the Rowland grating, which was necessary to establish experimentally the existence of the $m T$ series as discussed below. The stronger lines are considerably overexposed in the case of the longer exposures and, in the work with the Rowland grating, show from 1 to 8 orders of Rowland ghosts. It is believed that the spectrum has been completely recorded in the region studied. Radiometric investigation of still longer waves is highly desirable.

\section{WAVE-LENGTH MEASUREMENTS}

The wave-length measurements have been maderelative to the international standards in the iron arc spectrum. The results from each spectrogram were brought to the scale of the interference measurements by applying a correction where a systematic difference was noted. While such corrections were usually of the order of from 0.01 to $0.02 \mathrm{~A}$, it was found necessary to apply a correction as great as $0.1 \mathrm{~A}$ in the region above $9,000 \mathrm{~A}$. The cause of this displacement between the xenon, and second order comparison spectrum of iron, too great to be accounted for by atmospheric dispersion, appears to lie in some unexplained temperature displacement of the plate or mounting during prolonged exposure. Wave lengths which have been determined by interference methods are given to thousandths or tenthousandths of an angstrom unit and can thus be distinguished from those lines for which only prism or grating measurements are available. The vacuum wave numbers corresponding to the wave lengths determined by interference methods have been computed directly from the atmospheric dispersion formula of Meggers and Peters. ${ }^{8}$ The use of spectrographs of higher dispersion and resolving power, together with

7 C. E. K. Mees, J. Opt. Soc. Am., vol. 22, p. 204, 1932.

W. F. Meggers and C. G. Peters, B. S. Sci. papers, vol. 14 (S. P. 327), p. 722; 1918. 
the application of interference methods to one-fourth of the lines, has not only greatly increased the accuracy of the wave-length determinations, but has made possible the resolution of nearly all close pairs of lines. Such pairs occur at $6,111,6,163,6,206,6,840,7,584,7,642$, $7,664,8,348,8,952$, and $9,374 \mathrm{~A}$. In a few cases, notably $7,119 \mathrm{~A}$, lines formerly regarded as double for purposes of classification are now shown to be single.

The list of Xe I wave lengths as finally adopted is presented in Table 1. After eliminating known lines due to Xe II, faint unclassified lines of doubtful origin, and identifiable Rowland ghosts, we have retained 538 lines. The real lines were in practically all cases distinguished from false lines or ghosts by repeated observations with different gratings. With but 13 exceptions, all of the lines are accounted for by combinations of identified spectral terms. The calculated values of the wave numbers of all classified lines, computed from the experimental terms, are also given in Table 1, and indicate the degree of accuracy of the wave-length determinations.

TABLE 1.-List of $X e I$ lines

\begin{tabular}{|c|c|c|c|c|c|c|c|c|c|}
\hline $\begin{array}{l}\text { Inten- } \\
\text { sity }\end{array}$ & $\begin{array}{l}\text { Wave } \\
\text { length }\end{array}$ & $\begin{array}{c}\text { Wave } \\
\text { number } \\
\text { observed }\end{array}$ & $\underset{\text { tion }}{\text { Combina- }}$ & $\begin{array}{l}\text { Wave } \\
\text { num- } \\
\text { ber } \\
\text { calcu- } \\
\text { lated }\end{array}$ & $\begin{array}{l}\text { Inten- } \\
\text { sity }\end{array}$ & $\begin{array}{l}\text { Wave } \\
\text { length }\end{array}$ & $\begin{array}{c}\text { Wave } \\
\text { number } \\
\text { observed }\end{array}$ & $\underset{\text { tion }}{\text { Combina- }}$ & $\begin{array}{l}\text { Wave } \\
\text { num- } \\
\text { ber } \\
\text { calcu- } \\
\text { lated }\end{array}$ \\
\hline $\begin{array}{l}1- \\
1- \\
1 h- \\
1 h- \\
1 h\end{array}$ & $\begin{array}{l}3,340.04 \\
3,348.63 \\
3,358.17 \\
3,358.96 \\
3,370.34\end{array}$ & $\begin{array}{l}29,931.18 \\
29,854.41 \\
29,769.60 \\
29,762.60 \\
29,662.11\end{array}$ & $\begin{array}{l}1 s_{5}-11 p_{8} \\
1 s_{5}-110 \\
1 s_{5}-10 p_{6} \\
1 s_{5}-10 p_{8} \\
1 s_{5}-10 \mathrm{U}\end{array}$ & $\begin{array}{r}1.2 \\
3.97 \\
.60 \\
.60 \\
1.95\end{array}$ & $\begin{array}{r}1 \\
10 \\
4 \\
3 \\
40\end{array}$ & & $\begin{array}{l}26,714.54 \\
26,692.00 \\
26,689.79 \\
26,336.42 \\
26,333.99\end{array}$ & $\begin{array}{l}1 s_{4}-6 \mathrm{~V} \\
1 s_{4}-6 \mathrm{Y} \\
1 s_{4}-6 \mathrm{X} \\
1 s_{5}-5 \mathrm{~V} \\
1 s_{5}-5 \mathrm{U}\end{array}$ & $\begin{array}{l}.752 \\
1.95 \\
.750 \\
.437 \\
.95\end{array}$ \\
\hline $\begin{array}{l}1 \\
1 \\
2 \\
1- \\
2\end{array}$ & $\begin{array}{l}3,383.20 \\
3,384.36 \\
3.400 .07 \\
3,400.79 \\
3,418.37\end{array}$ & $\begin{array}{l}29,540.36 \\
29,539.23 \\
29,402.75 \\
29,396.53 \\
29,245.35\end{array}$ & $\begin{array}{l}1 s_{5}-9 p_{6} \\
1 s_{5}-9 p_{8} \\
1 s_{5}-9 \mathrm{U} \\
1 s_{5}-9 \mathrm{Y} \\
1 s_{5}-8 p_{6}\end{array}$ & $\begin{array}{r}.36 \\
.23 \\
.19 \\
5.73 \\
.35\end{array}$ & $\begin{array}{r}30 \\
3 \\
30 \\
10 \\
15\end{array}$ & $\begin{array}{l}3,801.39 \\
3,801.90\end{array}$ & & $\begin{array}{l}1 s_{5}-5 \mathrm{Y} \\
1 s_{5}-5 \mathrm{X} \\
1 s_{4}-5 p_{5} \\
1 s_{4}-5 p_{6} \\
1 s_{4}-5 p_{7}\end{array}$ & $\begin{array}{l}.717 \\
.152 \\
.35 \\
.09 \\
.70\end{array}$ \\
\hline $\begin{array}{l}2 \\
3 \\
1 \\
4 \\
4\end{array}$ & $\begin{array}{l}3,420.60 \\
3,442.66 \\
3,443.83 \\
3,469.81 \\
3,472.36\end{array}$ & $\begin{array}{l}29,231.41 \\
29,039.01 \\
29,029.15 \\
28,811.80 \\
28,790.64\end{array}$ & $\begin{array}{l}1 s_{5}-8 p_{8} \\
1 s_{5}-8 \mathrm{U} \\
1 s_{5}-8 \mathrm{Y} \\
1 s_{5}-7 p_{6} \\
1 s_{5}-7 p_{8}\end{array}$ & $\begin{array}{r}.41 \\
8.77 \\
8.77 \\
.86 \\
.64\end{array}$ & $\begin{array}{r}2 \\
2 \\
60 \\
10 \\
120\end{array}$ & $\begin{array}{l}3,835.6 \\
3,942.29 \\
3,948.163 \\
3,948.72 \\
3,950.925\end{array}$ & $\begin{array}{l}26,064.2 \\
25,358.81 \\
25,321.095 \\
25,317.52 \\
25,303.393\end{array}$ & $\begin{array}{l}1 s_{4}-5 p_{9} \\
1 s_{-}-5 \mathrm{~V} \\
1 s_{4}-5 \mathrm{Y} \\
1 s_{-}-5 \mathrm{X} \\
1 s_{6}-4 p_{6}\end{array}$ & $\begin{array}{l}5.00 \\
.821 \\
.101 \\
.536 \\
.395\end{array}$ \\
\hline $\begin{array}{l}1 \\
5 \\
2 \\
2 \\
2\end{array}$ & $\begin{array}{l}3,496.86 \\
3,506.74 \\
3,508.42 \\
3,517.90 \\
3,533.48\end{array}$ & $\begin{array}{l}28,588.93 \\
28,508.39 \\
28,494.74 \\
28,417.95 \\
28,292.66\end{array}$ & $\begin{array}{l}1 s_{4}-9 p_{5} \\
1 s_{5}-7 \mathrm{U} \\
1 s_{5}-7 \mathrm{Y} \\
1 s_{4}-9 \mathrm{Y} \\
1 s_{4}-8 p_{5}\end{array}$ & $\begin{array}{c}.93 \\
.43 \\
5.032 \\
8.12 \\
.66\end{array}$ & $\begin{array}{r}6 \\
200 \\
40 \\
30 \\
100\end{array}$ & & $\begin{array}{l}25,265.50 \\
25,197.423 \\
25,153.835 \\
25,085.762 \\
24,509.996\end{array}$ & & $\begin{array}{l}.541 \\
.423 \\
.837 \\
.762 \\
.996\end{array}$ \\
\hline $\begin{array}{c}1- \\
1 \\
10 \\
10 \\
1\end{array}$ & $\begin{array}{l}3,536.61 \\
3,537.35 \\
3,549.86 \\
3,554.04 \\
3,555.92\end{array}$ & & & $\begin{array}{l}.74 \\
.70 \\
.05 \\
.99 \\
.12\end{array}$ & $\begin{array}{r}60 \\
80 \\
20 \\
2 \\
20\end{array}$ & $\begin{array}{l}4,109.7093 \\
4,116.1151 \\
4,135.1337 \\
4,146.78 \\
4,193.01\end{array}$ & $\begin{array}{l}24,325.782 \\
24,287.925 \\
24,176.222 \\
24,108.32 \\
23,842.52\end{array}$ & $\begin{array}{l}1 s_{4}-4 p_{6} \\
1 s_{4}-4 p_{7} \\
1 s_{4}-4 p_{9} \\
1 s_{4}-4 p_{10} \\
1 s_{5}-4 \mathrm{~V}\end{array}$ & $\begin{array}{l}.779 \\
.925 \\
.221 \\
.146 \\
.536\end{array}$ \\
\hline $\begin{array}{r}3 \\
4 \\
1 \\
2 \\
15\end{array}$ & $\begin{array}{l}3,563.80 \\
3,587.02 \\
3,591.67 \\
3,592.80 \\
3,610.32\end{array}$ & $\begin{array}{l}28,051.95 \\
27,870.37 \\
27,834.29 \\
27,825.53 \\
27,690.51\end{array}$ & $\begin{array}{l}1 s_{4}-8 Y \\
1 s_{4}-7 p_{5} \\
1 s_{4}-7 p_{6} \\
1 s_{4}-7 p_{7} \\
1 s_{5}-6 U\end{array}$ & $\begin{array}{r}2.16 \\
.37 \\
.25 \\
.53 \\
.53\end{array}$ & $\begin{array}{r}150 \\
50 \\
10 \\
20 \\
100\end{array}$ & $\begin{array}{l}4,193.5296 \\
4,203.6945 \\
4,205.404 \\
4,372.287 \\
4,383,9092\end{array}$ & $\begin{array}{l}23,839.567 \\
23,781.922 \\
23,772.254 \\
22,864.925 \\
22,804.306\end{array}$ & & $\begin{array}{l}.567 \\
.921 \\
.253 \\
.920 \\
.305\end{array}$ \\
\hline $\begin{array}{r}8 \\
6 \\
10 \\
2 \\
4\end{array}$ & $\begin{array}{l}3,613.06 \\
3,633.06 \\
3,669.91 \\
3,677.54 \\
3,679.31\end{array}$ & $\begin{array}{l}27,669.50 \\
27,517.19 \\
27,240.90 \\
27,184.38 \\
27,171.30\end{array}$ & $\begin{array}{l}1 s_{1}-7 \mathrm{Y} \\
1 s_{1}-6 p \\
1 s_{4}-6 p \\
1 s_{4}-6 p\end{array}$ & \begin{tabular}{l|}
.56 \\
.416 \\
.90 \\
.44 \\
.30
\end{tabular} & $\begin{array}{r}70 \\
500 \\
400 \\
2 \\
300\end{array}$ & $\begin{array}{l}4,385.7693 \\
4,500.9772 \\
4,524.6805 \\
4,576.60 \\
4,582.7474\end{array}$ & $\begin{array}{l}22,794.635 \\
22,211.188 \\
22,094.833 \\
21,844.18 \\
21,814.878\end{array}$ & $\begin{array}{l}1 s_{s}-2 p_{2} \\
1 s_{5}-2 p_{3} \\
1 s_{4}-2 p_{1}\end{array}$ & $\begin{array}{l}.637 \\
.186 \\
.833 \\
.875\end{array}$ \\
\hline $\begin{array}{r}40 \\
1 \\
40 \\
4 \\
2\end{array}$ & $\begin{array}{l}3,685.90 \\
3,688.80 \\
3,693.49 \\
3,696.82 \\
3,702.74\end{array}$ & $\begin{array}{l}27,122.72 \\
27,101.40 \\
27,066.99 \\
27,042.61 \\
26,999.37\end{array}$ & $\begin{array}{l}1 s_{6}-5 p_{6} \\
1 s_{5}-5 p_{7} \\
1 s_{5}-5 p_{8} \\
1 s_{5}-5 p_{9} \\
1 s_{5}-5 p_{10}\end{array}$ & $\begin{array}{l}.70 \\
.32 \\
.99 \\
.61 \\
.41\end{array}$ & $\begin{array}{r}100 \\
1,000 \\
2,000 \\
100 \\
300\end{array}$ & $\begin{array}{l}4,611.8896 \\
4,624.2757 \\
4,671.226 \\
4,690.9711 \\
4,697.020\end{array}$ & $\begin{array}{l}21,677.034 \\
21,618.973 \\
21,401.685 \\
21,311.603 \\
21,284.155\end{array}$ & $\begin{array}{l}1 s s-3 p_{7} \\
1 s-3 p_{6} \\
1 s_{s}-3 p_{8} \\
1 s_{s}-2 p_{4} \\
1 s_{5}-3 p_{0}\end{array}$ & $\begin{array}{l}.034 \\
.973 \\
.685 \\
.600 \\
.154\end{array}$ \\
\hline
\end{tabular}


TABLE 1.-List of $\mathrm{X} e \mathrm{I}$ lines-Continued

\begin{tabular}{|c|c|c|c|c|c|c|c|c|c|}
\hline $\begin{array}{l}\text { Inten- } \\
\text { sity }\end{array}$ & $\begin{array}{l}\text { Ware } \\
\text { length }\end{array}$ & $\begin{array}{c}\text { Ware } \\
\text { number } \\
\text { observed }\end{array}$ & $\underset{\text { tion }}{\text { Combina- }}$ & $\begin{array}{l}\text { Ware } \\
\text { num- } \\
\text { ber } \\
\text { calcu- } \\
\text { lated }\end{array}$ & $\begin{array}{l}\text { Inten- } \\
\text { sity }\end{array}$ & $\begin{array}{l}\text { Wave } \\
\text { length }\end{array}$ & $\begin{array}{c}\text { Ware } \\
\text { number } \\
\text { otuserved }\end{array}$ & $\begin{array}{c}\text { Combina- } \\
\text { tion }\end{array}$ & $\begin{array}{l}\text { Ware } \\
\text { num- } \\
\text { ber } \\
\text { calcu- } \\
\text { lated }\end{array}$ \\
\hline $\begin{array}{r}5 \\
600 \\
150 \\
500 \\
400\end{array}$ & $\begin{array}{l}4,708.21 \\
4,734.1524 \\
4,792.6192 \\
4,807.019 \\
4,829.709\end{array}$ & $\begin{array}{l}21,233.57 \\
21,117.217 \\
20,859.605 \\
20,797.118 \\
20,699.415\end{array}$ & $\begin{array}{l}1 s_{4}-2 p_{2} \\
1 s_{4}-2 p_{3} \\
1 s_{5}-3 p_{10} \\
1 s_{4}-3 p_{5} \\
1 s_{4}-3 p_{7}\end{array}$ & $\begin{array}{r}.570 \\
.217 \\
.605 \\
.118 \\
.418\end{array}$ & $\begin{array}{r}3 \\
15 \\
80 \\
1 \\
5\end{array}$ & $\begin{array}{l}5,607.99 \\
5,612.65 \\
5,618.878 \\
5,621.24 \\
5,646.19\end{array}$ & $\begin{array}{l}17,826.76 \\
17,811.96 \\
17,792.220 \\
17,784.74 \\
17,706.16\end{array}$ & $\begin{array}{l}2 p_{\theta}-8 d_{1}^{\prime}{ }^{\prime} \\
2 p_{0}-8 d_{1}^{\prime \prime} \\
2 p_{0}-8 d_{4} \\
2 p_{p}-8 d_{3} \\
2 p_{8}-7 s_{5}\end{array}$ & $\begin{array}{l}.820 \\
2.01 \\
.220 \\
.805 \\
.17\end{array}$ \\
\hline $\begin{array}{c}300 \\
500 \\
500 \\
3 h \\
200\end{array}$ & $\begin{array}{l}4,843.294 \\
4,916.508 \\
4,923.1522 \\
5,023.88 \\
5,028.2796\end{array}$ & $\begin{array}{l}20,641.357 \\
20,333.981 \\
20,306.538 \\
19,899.40 \\
19,881.989\end{array}$ & $\begin{array}{l}1 s_{4}-3 p_{6} \\
1 s_{4}-2 p_{4} \\
1 s_{4}-3 p_{9} \\
1 s_{3}-8 \overline{\mathrm{X}} \\
1 s_{4}-3 p_{10}\end{array}$ & $\begin{array}{l}.357 \\
.984 \\
.538 \\
.47 \\
.989\end{array}$ & $\begin{array}{c}2 h \\
1 h- \\
1 h \\
40 \\
100\end{array}$ & $\begin{array}{l}5,6.72 .84 \\
5,654.31 \\
5,664.46 \\
5,688.373 \\
5,695.750\end{array}$ & $\begin{array}{l}17,685.33 \\
17,680.73 \\
17,649.05 \\
17,574.855 \\
17,552.094\end{array}$ & $\begin{array}{l}2 p_{7}-10 d_{1}^{\prime \prime} \\
2 p_{0}-8 d_{6} \\
2 p_{6}-11 d_{1}^{\prime} \\
1 s_{2}-6 \mathrm{~V} \\
1 s_{2}-6 \mathrm{Y}\end{array}$ & $\begin{array}{l}.31 \\
.77 \\
.05 \\
.855 \\
.094\end{array}$ \\
\hline $\begin{array}{c}10 \\
1 h \\
1 h \\
2 h \\
1\end{array}$ & $\begin{array}{l}5,162.711 \\
5,164.39 \\
5,167.30 \\
5,185.85 \\
5,206.07\end{array}$ & $\begin{array}{l}19,364.290 \\
19,357.99 \\
19,347.09 \\
19,277.89 \\
19,203.01\end{array}$ & $\mid \begin{array}{r}1 s_{3}-7 \mathrm{X} \\
2 p_{10}-10 d_{3} \\
2 p_{10}-10 d_{5} \\
1 s_{2}-9 \mathrm{Y} \\
2 p_{10}-8 s_{8}\end{array}$ & $\begin{array}{l}.290 \\
.94 \\
.08 \\
8.22 \\
2.99\end{array}$ & $\begin{array}{c}80 \\
8 \\
1 \\
3 \\
10 h\end{array}$ & & $\begin{array}{l}17,549.845 \\
17,543.50 \\
17,528.73 \\
17,517.89 \\
17,508.90\end{array}$ & $\begin{array}{c}1 s_{2}-6 \delta \\
2 p_{8}-8 d_{1}^{\prime} \\
2 p_{8}-8 d_{1}{ }^{\prime \prime} \\
2 p_{10}-5 \varepsilon_{4} \\
2 p_{\varepsilon}-8 d_{4}\end{array}$ & $\begin{array}{l}.851 \\
.561 \\
.75 \\
.954 \\
.961\end{array}$ \\
\hline $\begin{array}{l}4 h \\
4 h \\
2 h \\
1 h \\
2 h\end{array}$ & $\begin{array}{l}5,245.27 \\
5,248.98 \\
5,251.89 \\
5,273.48 \\
5,283.30\end{array}$ & $\begin{array}{l}19,059.51 \\
19,046.03 \\
19,035.48 \\
18,957.55 \\
18,922.31\end{array}$ & $\begin{array}{c}2 p_{10}-9 d_{3} \\
2 p_{10}-9 d_{5} \\
1 s_{3}-6 p_{10} \\
1 s_{2}-8 \mathrm{~V}\end{array}$ & $\begin{array}{l}.48 \\
.02 \\
.59 \\
.31\end{array}$ & $\begin{array}{l}2 \\
70 \\
80 \\
15 h \\
1 h\end{array}$ & & $\begin{array}{l}17,501.52 \\
17,490.780 \\
17,489.140 \\
17,471.15 \\
17,467.72\end{array}$ & $\begin{array}{c}2 p_{\varepsilon}-8 d_{3} \\
2 p_{10} 5 s_{5} \\
2 p_{8}-8 d_{4}^{\prime} \\
2 p_{6}-6 s_{4} \\
2 p_{7}-9 d_{2}\end{array}$ & $\begin{array}{l}.546 \\
.783 \\
.140 \\
.18 \\
.74\end{array}$ \\
\hline $\begin{array}{l}4 h \\
3 h \\
3 \\
1 h- \\
2 h\end{array}$ & $\begin{array}{l}5,286.11 \\
5,286.38 \\
5,306.37 \\
5,335.91 \\
5,337.89\end{array}$ & $\begin{array}{l}18,912.25 \\
18,911.29 \\
18,840.05 \\
18,735.75 \\
18,728.80\end{array}$ & $\left\{\begin{array}{c}1 s_{2}-8 \mathrm{Y} \\
1 s_{2}-8 \mathrm{X} \\
2 p_{10-} 7 s_{5} \\
2 p_{q}-11 d_{1}^{\prime \prime} \\
2 p_{q}-11 d_{4} \\
2 p_{8}-13 d_{4}^{\prime}\end{array}\right.$ & $\begin{array}{l}.26 \\
.20 \\
.08 \\
.78 \\
.80\end{array}$ & $\begin{array}{l}4 \\
4 h \\
6 \\
1 h \\
8 h\end{array}$ & $\begin{array}{l}5,726.10 \\
5,733.48 \\
5,740.17 \\
5,740.73 \\
5,748.20\end{array}$ & $\begin{array}{l}17,459.06 \\
17,436.59 \\
17,416.27 \\
17,414.57 \\
17,391.94\end{array}$ & $\begin{array}{c}2 p_{\theta}-6 s_{5} \\
2 p_{6}-10 d_{1}{ }^{\prime} \\
2 p_{10}-6 d_{2} \\
2 p_{6}-10 d_{3} \\
2 p_{7}-9 d_{1}{ }^{\prime \prime}\end{array}$ & $\begin{array}{r}.131 \\
.60 \\
.29 \\
.62 \\
2.00\end{array}$ \\
\hline $\begin{array}{c}1 \\
15 \\
30 \\
6 \\
1 h-\end{array}$ & $\begin{array}{l}5,356.80 \\
5,362.244 \\
5,364.626 \\
5,367.03 \\
5,373.74\end{array}$ & $\begin{array}{l}18,662.68 \\
18,643.739 \\
18,635.459 \\
18,627.11 \\
18,603.85\end{array}$ & $\begin{array}{l}2 p_{10}-8 d_{1}^{\prime \prime} \\
2 p_{10}-\delta d_{5} \\
2 p_{10}-8 d_{3} \\
2 p_{\delta}-12 d_{4}^{\prime}\end{array}$ & $\begin{array}{l}.66 \\
.739 \\
.459 \\
.85\end{array}$ & $\begin{array}{l}1 h \\
1 h \\
15 \\
60 \\
25\end{array}$ & $\begin{array}{l}5,754.60 \\
5,792.26 \\
5,807.311 \\
5,814.505 \\
5,820.52\end{array}$ & $\begin{array}{l}17,372.59 \\
17,259.64 \\
17,214.912 \\
17,193.614 \\
17,175.84\end{array}$ & 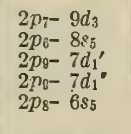 & $\begin{array}{l}.59 \\
.67 \\
.922 \\
.616 \\
.907\end{array}$ \\
\hline $\begin{array}{r}100 \\
20\end{array}$ & $\begin{array}{l}5,392.795 \\
5,394.738\end{array}$ & $\begin{array}{l}18,538.119 \\
18,531.443\end{array}$ & $\begin{array}{r}1 s_{3}-6 \mathrm{X} \\
2 p_{10}-8 d_{6}\end{array}$ & $\begin{array}{l}.119 \\
.443\end{array}$ & $\begin{array}{l}300 \\
150\end{array}$ & & $\begin{array}{l}17,165.906 \\
17,163.225\end{array}$ & $\begin{array}{r}1 s_{3}-5 \mathrm{X} \\
2 p_{8}-7 d_{4}\end{array}$ & $\begin{array}{l}.907 \\
.225\end{array}$ \\
\hline $1 h$ & $5,397.63$ & $18,521.51$ & & & $\begin{array}{c}1 \\
20 h \\
4 h\end{array}$ & $\begin{array}{l}5,8 \\
5,8 \\
5,8\end{array}$ & $\begin{array}{l}17,154.63 \\
17,146.06 \\
17,16.12\end{array}$ & $\begin{array}{l}2 p_{0}-7 d_{3} \\
2 p_{6}-9 d_{1}^{\prime} \\
2 p_{6}-9 d_{3}\end{array}$ & $\begin{array}{l}.636 \\
.10 \\
16\end{array}$ \\
\hline $\begin{array}{l}4 h \\
5\end{array}$ & $\begin{array}{l}5,400.45 \\
5,418.02\end{array}$ & $\begin{array}{l}18,511.84 \\
18,451.81\end{array}$ & $2 p_{0}$ & & 5 & $5,840.83$ & $17,116.12$ & $\begin{array}{l}2 p_{6}-9 d_{3} \\
2 p_{9}-7 d_{6}\end{array}$ & .610 \\
\hline $\begin{array}{c}2 h \\
5 h \\
30 \\
15 \\
1 h\end{array}$ & $\begin{array}{l}5,421.76 \\
5,435.60 \\
5,439.923 \\
5,440.39 \\
5,444.87\end{array}$ & \begin{tabular}{|l|}
$18,439.08$ \\
$18,392.13$ \\
$18,377.519$ \\
$18,375.94$ \\
$18,360.82$
\end{tabular} & $\begin{array}{c}2 p_{8}-11 d_{4}{ }^{\prime} \\
1 s_{2}-7 \mathrm{~V} \\
1 s_{2}-7 \mathrm{Y} \\
1 s_{2}-7 \mathrm{X} \\
2 p_{0}-8 s_{4}\end{array}$ & $\begin{array}{c}.08 \\
.13 \\
.519 \\
6.022 \\
.82\end{array}$ & $\begin{array}{c}1 \\
2 \\
3 h \\
15\end{array}$ & $\begin{array}{l}5,845.46 \\
5,846.21 \\
5,849.85 \\
5,856.509\end{array}$ & $\begin{array}{l}17,102.56 \\
17,100.37 \\
17,089.73 \\
17,070.300\end{array}$ & $\begin{array}{c}2 p_{6}-9 d_{8} \\
1 s_{2}-5 p_{5} \\
2 p_{7}-8 d_{2} \\
2 p_{10}-6 d_{1}\end{array}$ & $\begin{array}{l}.70 \\
.45 \\
.74 \\
.300\end{array}$ \\
\hline $\begin{array}{c}1 h \\
2 \\
15 \\
1 h \\
1 h\end{array}$ & $\begin{array}{l}5,454.54 \\
5,456.45 \\
5,460.037 \\
5,479.12 \\
5,481.33\end{array}$ & $\begin{array}{l}18,328.27 \\
18,321.85 \\
18,309.820 \\
18,246.05 \\
18,238.69\end{array}$ & $\begin{array}{l}2 p_{8}-9 s_{5} \\
2 p_{10}-6 s_{4} \\
2 p_{10}-6 s_{5} \\
2 p_{8}-10 d_{1}^{\prime} \\
2 p_{9}-9 d_{1}^{\prime}\end{array}$ & $\begin{array}{l}.27 \\
.83 \\
.820 \\
.01 \\
.77\end{array}$ & $\begin{array}{r}100 \\
6 \\
20 \\
100 \\
2 h\end{array}$ & $\begin{array}{l}5,875.018 \\
5,878.92 \\
5,889.12 \\
5,894.988 \\
5,895.62\end{array}$ & $\begin{array}{l}17,016.519 \\
17,005.23 \\
16,975.77 \\
16,958.874 \\
16,957.05\end{array}$ & $\begin{array}{c}2 p_{10}-6 d_{3} \\
1 s_{2}-5 p_{6} \\
2 p_{7}-8 d_{1} \\
2 p_{10}-6 d_{5} \\
2 p_{7}-8 d_{5}\end{array}$ & $\begin{array}{l}.519 \\
.19 \\
.77 \\
.874 \\
6.850\end{array}$ \\
\hline $\begin{array}{r}1 h \\
4 h \\
6 h \\
20 h \\
2 h\end{array}$ & $\begin{array}{l}5,484.16 \\
5,484.46 \\
5,487.03 \\
5,488.555 \\
5,532.78\end{array}$ & $\begin{array}{l}18,229.28 \\
18,228.28 \\
18,219.75 \\
18,214.684 \\
18,060.10\end{array}$ & $\begin{array}{l}2 p_{8}-10 d_{4} \\
2 p_{8}-9 d_{1}^{\prime \prime} \\
2 p_{8}-10 d_{4}^{\prime} \\
2 p_{9}-9 d_{4} \\
2 p_{8}-8 s_{5}\end{array}$ & $\begin{array}{l}8.60 \\
.24 \\
.75 \\
.684 \\
.08\end{array}$ & $\begin{array}{r}8 \\
20 \\
3 \\
5 \\
4\end{array}$ & $\begin{array}{l}5,898.56 \\
5,904.462 \\
5,906.76 \\
5,911.90 \\
5,916.65\end{array}$ & $\begin{array}{l}16,948.60 \\
16,931.663 \\
16,925.07 \\
16,910.36 \\
16,896.78\end{array}$ & $\begin{array}{l}2 p_{7}-8 d_{3} \\
2 p_{\delta}-7 d_{1}^{\prime} \\
1 s_{2}-5 p_{0^{\prime}} \\
2 p_{\delta^{-}} 7 d_{1}{ }^{\prime} \\
2 p_{0^{-}}-7 s_{5}\end{array}$ & $\begin{array}{l}.570 \\
.663 \\
.10 \\
.357 \\
.76\end{array}$ \\
\hline $3 h$ & $5,540.38$ & $18,044.30$ & $\left\{\begin{array}{c}1 s_{2}-6 p_{6} \\
2 p_{10}-7 d_{1}^{\prime \prime}\end{array}\right.$ & $\begin{array}{l}.24 \\
.270\end{array}$ & $\begin{array}{l}10 \\
20\end{array}$ & 50 & $\begin{array}{l}95 \\
.953 \\
38\end{array}$ & $\begin{array}{l}18_{2-} \\
2 p_{8-} \\
2 p^{-}\end{array}$ & $\begin{array}{l}.90 \\
.966 \\
377\end{array}$ \\
\hline $\begin{array}{l}\text { So } \\
3 h \\
1-\end{array}$ & $\begin{array}{l}5,552.385 \\
5,553.10 \\
5,555.06\end{array}$ & $\begin{array}{l}18,005.291 \\
18,002.97 \\
17,996.62\end{array}$ & $\begin{array}{c}2 p_{10}-7 d_{3} \\
2 p_{s}-7 s_{4} \\
1 s_{2}-6 p_{0}\end{array}$ & $\begin{array}{l}.291 \\
.97 \\
.61\end{array}$ & $\begin{array}{r}6 \\
80 \\
100\end{array}$ & $\begin{array}{l}5,925.56 \\
5,931.241 \\
5,934.172\end{array}$ & $\begin{array}{l}16,871.38 \\
16, \$ 55.219 \\
16,846.894\end{array}$ & $\begin{array}{c}2 p_{5}-7 d_{3} \\
2 p_{10}-6 d_{6} \\
2 p_{8}-7 d_{4}^{\prime}\end{array}$ & $\begin{array}{l}.377 \\
.220 \\
.894\end{array}$ \\
\hline $\begin{array}{c}2 \\
2 \\
5 h \\
100 \\
2 h\end{array}$ & $\begin{array}{l}5,557.28 \\
5,563.50 \\
5,566.22 \\
5,566.615 \\
5,567.77\end{array}$ & $\begin{array}{l}17,989.43 \\
17,969.32 \\
17,960.54 \\
17,959.264 \\
17,955.54\end{array}$ & $\begin{array}{c}2 p_{8}-7 s_{5} \\
1 s_{2}-6 p_{10} \\
2 p_{10}-7 d_{5} \\
2 p_{8}-9 d_{1}^{\prime}\end{array}$ & .264 & $\begin{array}{c}1- \\
1 h \\
40 \\
2 \\
1\end{array}$ & $\begin{array}{l}5,970.41 \\
5,972.82 \\
5,974.152 \\
5,978.29 \\
5,979.42\end{array}$ & $\begin{array}{l}16,744.64 \\
16,737.88 \\
16,734.153 \\
16,722.57 \\
16,719.41\end{array}$ & $\begin{array}{l}3 d_{b}-10 \mathrm{~V} \\
3 d_{b^{\prime}}-10 \mathrm{X}, \mathrm{Y} \\
2 p_{6}-8 d_{1}^{\prime} \\
3 d_{4^{\prime}-11 \mathrm{~T}}, \mathrm{Z} \\
2 p_{6}-8 d_{1}{ }^{{ }^{2}}\end{array}$ & $\begin{array}{l}.64 \\
.153 \\
.57 \\
.34\end{array}$ \\
\hline $\begin{array}{l}2 h \\
40 \\
50 \\
1- \\
6\end{array}$ & $\begin{array}{l}5,575.27 \\
5,579.28 \\
5,581.784 \\
5,585.18 \\
5,594.37\end{array}$ & $\begin{array}{l}17,931.38 \\
17,918.50 \\
17,910.458 \\
17,899.57 \\
17,870.16\end{array}$ & $\begin{array}{c}2 p_{8}-9 d_{4} \\
2 p_{8}-9 d_{4}^{\prime} \\
2 p_{10}-7 d_{6} \\
2 p_{7}-11 d_{1}^{\prime \prime} \\
1 s_{3}-5 p_{10}\end{array}$ & $\begin{array}{l}.058 \\
.54 \\
.54\end{array}$ & $\begin{array}{r}4 \\
20 \\
30 \\
15 \\
8\end{array}$ & $\begin{array}{l}5,986.23 \\
5,989.18 \\
5,998.115 \\
6,007.909 \\
6,009.78\end{array}$ & $\begin{array}{l}16,700.39 \\
16,692.16 \\
16,667.300 \\
16,640.128 \\
16,634.94\end{array}$ & $\begin{array}{l}2 p_{6}-8 d_{s} \\
2 p_{6}-8 d_{3} \\
2 p_{8}-5 s_{4} \\
2 p_{8}-5 s_{5} \\
2 p_{7}-6 s_{4}\end{array}$ & $\begin{array}{l}.418 \\
.138 \\
.300 \\
.128 \\
.94\end{array}$ \\
\hline
\end{tabular}

$148938-33-10$ 
TABLE 1.-List of $X e I$ lines-Continued

\begin{tabular}{|c|c|c|c|c|c|c|c|c|c|}
\hline $\begin{array}{l}\text { ten- } \\
\text { ity }\end{array}$ & $\begin{array}{l}\text { Wave } \\
\text { length }\end{array}$ & $\begin{array}{c}\text { Wave } \\
\text { number } \\
\text { observed }\end{array}$ & $\begin{array}{l}\text { Combina- } \\
\text { tion }\end{array}$ & $\begin{array}{l}\text { Wave } \\
\text { num- } \\
\text { ber } \\
\text { calcu- } \\
\text { lated }\end{array}$ & $\begin{array}{l}\text { Inten- } \\
\text { sity }\end{array}$ & $\begin{array}{l}\text { Wave } \\
\text { length }\end{array}$ & $\begin{array}{c}\text { Wave } \\
\text { number } \\
\text { observed }\end{array}$ & $\begin{array}{c}\text { Combina- } \\
\text { tion }\end{array}$ & $\begin{array}{l}\text { Wave } \\
\text { num- } \\
\text { ber } \\
\text { calcu- } \\
\text { lated }\end{array}$ \\
\hline $\begin{array}{l}1 h \\
1 h- \\
4 \\
1 h- \\
2\end{array}$ & $\begin{array}{l}6,014.10 \\
6,022.89 \\
6,026.76 \\
6,031.36 \\
6,034.92\end{array}$ & \begin{tabular}{|}
$16,623.00$ \\
$16,598.74$ \\
$16,588.03$ \\
$16,575.43$ \\
$16,565.65$
\end{tabular} & $\begin{array}{l}2 p_{7}-6 s_{5} \\
3 d_{3}-11 U \\
2 p_{6}-8 d_{6} \\
2 p_{5}-10 d_{2} \\
2 p_{\vartheta}-6 d_{2}\end{array}$ & $\begin{array}{l}2.931 \\
.74 \\
.10 \\
.46 \\
.64\end{array}$ & $\begin{array}{c}20 \\
2 h \\
7 \\
10 h l \\
3\end{array}$ & & \begin{tabular}{|l|}
$15,547.436$ \\
$15,502.72$ \\
$15,498.45$ \\
$15,495.30$ \\
$15,472.02$
\end{tabular} & $\begin{array}{l}2 p_{b}-5 s_{5} \\
3 d_{4}-9 \mathrm{~W} \\
2 p_{9}-2 s_{2} \\
3 d_{4}-9 Z \\
2 p_{5}-6 s_{4}\end{array}$ & $\begin{array}{l}.461 \\
.71 \\
.45 \\
.30 \\
.01\end{array}$ \\
\hline $\begin{array}{c}10 \\
6 h \\
1 h \\
2 h \\
1 h\end{array}$ & $\begin{array}{l}6,043.38 \\
6,048.00 \\
6,064.91 \\
6,067.52 \\
6,067.77\end{array}$ & $\begin{array}{l}16,542.46 \\
16,529.82 \\
16,483.74 \\
16,476.65 \\
16,475.97\end{array}$ & $\begin{array}{c}2 p_{7}-7 d_{2} \\
3 d_{4}^{\prime}-10 \mathrm{~T}, \mathrm{Z} \\
3 d_{5}-9 \mathrm{~V} \\
3 d_{5}-9 \mathrm{Y} \\
3 d_{5}-9 \mathrm{X}\end{array}$ & $\begin{array}{l}.45 \\
.82 \\
.74 \\
.62 \\
.98\end{array}$ & $\begin{array}{l}300 \\
150 \\
120 \\
30 h l \\
100\end{array}$ & & & $\begin{array}{l}2 p_{10}-5 d_{3} \\
2 p_{10}-5 d_{2} \\
2 p_{10}-5 d_{1}^{\prime \prime} \\
3 d_{1}^{\prime}-7 W^{\prime} \\
2 p_{7}-6 d_{1}^{\prime \prime}\end{array}$ & $\begin{array}{l}.394 \\
.906 \\
.383 \\
.48 \\
.411\end{array}$ \\
\hline $\begin{array}{c}3 \\
1- \\
3 \\
8 \\
30\end{array}$ & $\begin{array}{l}6,093.38 \\
6,095.15 \\
6,103.88 \\
6,108.37 \\
6,111.759\end{array}$ & \begin{tabular}{|l|}
$16,406.72$ \\
$16,401.96$ \\
$16,378.50$ \\
$16,366.46$ \\
$16,357.384$
\end{tabular} & $\begin{array}{l}3 d_{3}-10 \mathrm{U} \\
3 d_{3}-10 \mathrm{X}, \mathrm{Y} \\
2 p_{6}-6 s_{4} \\
2 p_{6}-6 s_{5} \\
2 p_{7}-7 d_{1}^{\prime \prime}\end{array}$ & \begin{tabular}{l|}
.72 \\
.51 \\
.519 \\
.381
\end{tabular} & $\begin{array}{c}15 \\
200 h \\
3 \\
40\end{array}$ & $\begin{array}{l}6,500.37 \\
6,504.18 \\
6,507.50 \\
6,521 \cdot 508\end{array}$ & \begin{tabular}{|l|}
$15,379.50$ \\
$15,370.49$ \\
$15,362.65$ \\
$15,329.648$
\end{tabular} & $\begin{array}{c}2 p_{5}-7 d_{2} \\
3 d_{4}^{\prime}-7 \mathrm{~T}, Z \\
1 s_{2}-4 p_{3}\end{array}$ & $\begin{array}{l}.52 \\
.49 \\
.10 \\
.630\end{array}$ \\
\hline $\begin{array}{r}40 \\
10 \\
5 \\
15 \\
1\end{array}$ & $\begin{array}{l}6,111.951 \\
6,114.86 \\
6,123.91 \\
6,126.36 \\
6,131.47\end{array}$ & $\begin{array}{l}16,356.870 \\
16,349.09 \\
16,324.93 \\
16,318.40 \\
16,304.80\end{array}$ & $\begin{array}{r}2 p_{8}-5 s_{5} \\
2 p_{10}-2 s_{2} \\
3 d_{6}-8 \mathrm{X} \\
2 p_{7}-7 d_{3} \\
2 p_{5}-9 d_{2}\end{array}$ & \begin{tabular}{l|}
.869 \\
.10 \\
.96 \\
.401 \\
.80
\end{tabular} & $\begin{array}{c}100 \\
40 \\
20 \\
4 \\
50 h l\end{array}$ & & \begin{tabular}{|l|}
$15,302.312$ \\
$15,278.456$ \\
$15,272.01$ \\
$15,254.45$ \\
$15,253.196$
\end{tabular} & $\begin{array}{l}2 p_{\vartheta}-4 s_{4} \\
2 p_{9}-4 s_{5} \\
2 p_{7}-6 d_{5} \\
3 d_{3}-7 \mathrm{~V} \\
3 d_{3}-7 \mathrm{U}\end{array}$ & $\begin{array}{l}.312 \\
.455 \\
1.985 \\
.43 \\
.20\end{array}$ \\
\hline $\begin{array}{c}1 h \\
4 \\
20 h w \\
20 \\
3\end{array}$ & $\begin{array}{l}6,142.13 \\
6,143.70 \\
6,144.97 \\
6,152.069 \\
6,162.16\end{array}$ & $\begin{array}{l}16,276.50 \\
16,272.34 \\
16,268.98 \\
16,250.206 \\
16,223.60\end{array}$ & $\begin{array}{l}3 d_{i^{\prime}}^{\prime}-9 \mathrm{~W} \\
2 p_{7}-7 d_{5} \\
3 d_{i^{\prime}}-9 \mathrm{~T}, \mathrm{Z} \\
2 p_{9}-6 d_{1}^{\prime} \\
2 p_{7}-7 d_{6}\end{array}$ & \begin{tabular}{l||}
.53 \\
.375 \\
.98 \\
.201 \\
.569
\end{tabular} & $\begin{array}{c}25 \\
4 h \\
20 \\
8 \\
100\end{array}$ & & \begin{tabular}{|l|}
$15,239.77$ \\
$15,238.19$ \\
$15,185.83$ \\
$15,168.35$ \\
$15,157.531$
\end{tabular} & & $\begin{array}{l}.80 \\
.30 \\
.882 \\
.331 \\
.534\end{array}$ \\
\hline $\begin{array}{r}90 \\
30 \\
150 \\
120 \\
300\end{array}$ & & \begin{tabular}{|r|}
$16,219.648$ \\
$16,218.924$ \\
$16,181.209$ \\
$16,177.640$ \\
$16,170.431$
\end{tabular} & $\begin{array}{c}2 p_{9}-6 d_{1}^{\prime \prime} \\
1 s_{2}-5 \mathrm{~V} \\
1 s_{2}-5 \mathrm{Y} \\
1 s_{2}-5 \mathrm{X} \\
2 p_{9}-6 d_{4}\end{array}$ & $\begin{array}{l}.646 \\
.924 \\
.204 \\
.639 \\
.432\end{array}$ & $\begin{array}{c}4 h \\
30 h \\
10 \\
2 \\
50\end{array}$ & & & $\begin{array}{l}3 d_{1}-8 \mathrm{~W} \\
3 d_{-}-8 Z \\
2 p_{-}-6 d_{1} \\
2 p_{0}-6 d_{4} \\
2 p_{6}-6 d_{3}\end{array}$ & $\begin{array}{c}.75 \\
.30 \\
6.979 \\
.765 \\
.198\end{array}$ \\
\hline $\begin{array}{c}3 \\
20 \\
4 h \\
1 h \\
1\end{array}$ & $\begin{array}{l}6,184.16 \\
6,189.10 \\
6,191.40 \\
6,193.89 \\
6,195.49\end{array}$ & \begin{tabular}{|l|}
$16,165.88$ \\
$16,152.98$ \\
$16,146.98$ \\
$16,140.49$
\end{tabular} & $\begin{array}{c}2 p_{9}-6 d_{3} \\
2 p_{10}-4 s_{4} \\
3 d_{3}-9 \mathrm{U} \\
3 d_{3}-9 \mathrm{Y} \\
1 s_{3}-4 p_{7}\end{array}$ & $\begin{array}{l}.865 \\
.966 \\
.96 \\
.50 \\
.296\end{array}$ & $\begin{array}{r}3 \\
20 \\
4 \\
60 \\
150\end{array}$ & & $\begin{array}{l}15,036.28 \\
15,015.57 \\
14,999.95 \\
14,995.196 \\
14,990.800\end{array}$ & $\begin{array}{c}1 s_{2}-4 p_{9} \\
2 p_{6}-6 d_{5} \\
2 p_{5}-4 s_{5} \\
2 p_{10}-5 d_{6}\end{array}$ & $\begin{array}{l}.324 \\
.553 \\
.196 \\
.800\end{array}$ \\
\hline $\begin{array}{c}100 \\
60 \\
3 h \\
6 h \\
4\end{array}$ & $\begin{array}{l}6,198.260 \\
6,200.890 \\
6,201.49 \\
6,205.35 \\
6,205.75\end{array}$ & \begin{tabular}{|l|}
$16,129.108$ \\
$16,122.266$ \\
$16,120.71$ \\
$16,110.68$ \\
$16,109.64$
\end{tabular} & & $\begin{array}{l}.109 \\
.255 \\
.71 \\
.66 \\
.60\end{array}$ & $\begin{array}{r}25 \\
20 \\
1 \\
200\end{array}$ & $\begin{array}{l}6,678.972 \\
6,681.036 \\
6,706.46 \\
6,728.008\end{array}$ & $\begin{array}{l}14,968.239 \\
14,963.615 \\
14,906.89 \\
14,859.146\end{array}$ & $\begin{aligned} 1 s_{2}-4 p_{10} \\
3 d_{6}-6 \mathrm{X} \\
3 d_{3}-6 p_{6} \\
2 p_{10-}-5 d_{5}\end{aligned}$ & $\begin{array}{r}.249 \\
.613 \\
.82 \\
8.148 \\
8.89\end{array}$ \\
\hline $\begin{array}{r}20 \\
3 \\
1 \\
10 \\
3\end{array}$ & $\begin{array}{l}6,206.297 \\
6,209.11 \\
6,220.84 \\
6,224.169 \\
6,242.09\end{array}$ & \begin{tabular}{|l|}
$16,108.220$ \\
$16,100.92$ \\
$16,070.56$ \\
$16,061.968$ \\
$16,015.85$
\end{tabular} & & $\begin{array}{l}.220 \\
.949 \\
.558 \\
.969 \\
.943\end{array}$ & $\begin{array}{l}10 \\
50 \\
40 \\
12 \\
15\end{array}$ & & $\begin{array}{l}14,773.27 \\
14,750.48 \\
14,748.25 \\
14,668.09 \\
14,662.20\end{array}$ & & $\begin{array}{l}.26 \\
.49 \\
.251 \\
.129 \\
.21\end{array}$ \\
\hline $\begin{array}{c}50 \\
40 \\
1 h \\
10 \\
4\end{array}$ & $\begin{array}{l}6,261.212 \\
6,265.301 \\
6,268.34 \\
6,273.23\end{array}$ & $\begin{array}{l}15,966.942 \\
15,956.522 \\
15,948.78\end{array}$ & $\begin{array}{l}2 p_{8}-6 d_{1}^{\prime} \\
183-4 p_{10} \\
3 d_{4}-11 Z \\
2 p_{8}-6 d_{1} \\
2 p_{5}-8 d_{2}\end{array}$ & $\begin{array}{l}.942 \\
.517 \\
.78 \\
.387 \\
.81\end{array}$ & $\begin{array}{r}200 \\
8 \\
20 \\
1 \\
2 h\end{array}$ & & $\begin{array}{l}14,643.014 \\
14,613.80 \\
14,612.65 \\
14,606.74 \\
14,605.52\end{array}$ & & $\begin{array}{l}.008 \\
.804 \\
.66 \\
.73 \\
.50\end{array}$ \\
\hline $\begin{array}{c}5 h \\
150 \\
50 \\
15 \\
15\end{array}$ & $\begin{array}{l}6,281.81 \\
6,256.011 \\
6,202.649 \\
6,291.45\end{array}$ & \begin{tabular}{|l|}
$15,914.58$ \\
$15,903.950$ \\
$15,887.173$ \\
$15,882.63$ \\
$15,831.02$
\end{tabular} & $\begin{array}{l}3 d_{t^{\prime}}-8 \mathrm{~W} \\
3 d_{i^{\prime}}-8 \mathrm{~T} \\
2 p_{8}-6 d_{4} \\
2 p_{5}-6 d_{3} \\
2 p_{7}-5 s\end{array}$ & $\begin{array}{l}.57 \\
.95 \\
.173 \\
.608 \\
.065\end{array}$ & $\begin{array}{l}60 \\
50 \\
30 \\
40 \\
20\end{array}$ & & & $\begin{array}{c}2 p_{9}-5 d_{3} \\
3 d_{4}-7 Z \\
2 p_{0}-5 d_{2} \\
3 d_{t}^{\prime}-6 \mathrm{~W} \\
2 p_{b}-6 d_{2}\end{array}$ & $\begin{array}{l}.740 \\
.05 \\
.252 \\
.82 \\
.47\end{array}$ \\
\hline $\begin{array}{c}500 \\
2 \\
20 \\
40 h l \\
8 / l l\end{array}$ & $\begin{array}{l}6,318.062 \\
6,325.81 \\
6,331.50 \\
6,333.97 \\
6,337.55\end{array}$ & \begin{tabular}{|l|}
$15,823.273$ \\
$15,803.89$ \\
$15,739.69$ \\
$15,783.53$ \\
$15,774.54$
\end{tabular} & $\begin{array}{l}2 p_{8}-6 d_{6}^{\prime} \\
2 p_{7}-585 \\
3 d_{6}-7 \mathrm{X} \\
3 d_{3}-8 \mathrm{U} \\
3 d_{3}-8 \mathrm{Y}\end{array}$ & $\begin{array}{l}.273 \\
.893 \\
.784 \\
.54 \\
.54\end{array}$ & $\begin{array}{r}5 \\
50 \\
100 \\
300 \\
30\end{array}$ & & $\begin{array}{l}14,561.40 \\
14,558.734 \\
14,547.571 \\
14,526.332 \\
14,466.08\end{array}$ & $\begin{array}{l}3 d_{4}^{\prime}-6 \mathrm{U} \\
2 p_{9}-5 d_{1}^{\prime \prime} \\
3 d_{4}^{\prime}-6 \mathrm{~T} \\
2 p_{q}-5 d_{4} \\
2 p_{\tau}-4 s_{i}\end{array}$ & $\begin{array}{l}.42 \\
.729 \\
.57 \\
.333 \\
.077\end{array}$ \\
\hline $\begin{array}{l}2 / 2 h \\
30 \\
10 \\
30\end{array}$ & $\begin{array}{l}6,344.98 \\
6,355.77 \\
0,412.38 \\
6,418.41\end{array}$ & \begin{tabular}{|l|}
$15,756.14$ \\
$15,729.40$ \\
$15,590.53$ \\
$15,575.89$
\end{tabular} & & $\begin{array}{l}.40 \\
.55 \\
.92 \\
.923\end{array}$ & $\begin{array}{r}8 \\
15 \\
100 \\
50\end{array}$ & $\begin{array}{l}6,924 \\
6,92\end{array}$ & & & $\begin{array}{l}.220 \\
.14 \\
.30 \\
.37\end{array}$ \\
\hline $33 / h$ & $0,418.95$ & $15,574.50$ & & & & $5,936.69$ & & & 3 \\
\hline
\end{tabular}


TABLE 1.-List of Xe I lines-Continued

\begin{tabular}{|c|c|c|c|c|c|c|c|c|c|}
\hline Inten- & $\begin{array}{l}\text { Wave } \\
\text { length }\end{array}$ & $\begin{array}{c}\text { Wave } \\
\text { number } \\
\text { observed }\end{array}$ & Combina- & $\begin{array}{l}\text { Wave } \\
\text { num- } \\
\text { ber } \\
\text { calcu- } \\
\text { lated }\end{array}$ & $\begin{array}{l}\text { Inten- } \\
\text { sity }\end{array}$ & $\begin{array}{l}\text { Wave } \\
\text { length }\end{array}$ & $\begin{array}{c}\text { Ware } \\
\text { number } \\
\text { observed }\end{array}$ & $\underset{\text { Combina- }}{\text { tion }}$ & $\begin{array}{l}\text { Ware } \\
\text { numi- } \\
\text { ber } \\
\text { calcu- } \\
\text { lated }\end{array}$ \\
\hline $\begin{array}{r}1 \\
100 \\
30 \\
1 \\
4\end{array}$ & $\begin{array}{l}6,949.76 \\
6,976.182 \\
6,982.05 \\
6,991.65 \\
7,003.10\end{array}$ & $\begin{array}{l}14,385.02 \\
14,330.542 \\
14,318.50 \\
14,298.84 \\
14,275.46\end{array}$ & $\begin{array}{l}2 p_{8}-5 d_{1}{ }^{\prime} \\
2 p_{8}-5 d_{3} \\
3 d_{5}-5 p_{6} \\
2 p_{8}-5 d_{1}^{\prime \prime}\end{array}$ & $\begin{array}{l}.545 \\
.481 \\
.85 \\
.470\end{array}$ & $\begin{array}{r}10 \\
1 \\
40 \\
50 \\
15\end{array}$ & $\begin{array}{l}7,666.61 \\
7,670.81 \\
7,740.31 \\
7,783.66 \\
7,789.42\end{array}$ & $\begin{array}{l}13,039.99 \\
13,032.85 \\
12,915.83 \\
12,843.90 \\
12,834.40\end{array}$ & $\begin{array}{c}3 d_{3}-5 \mathrm{X} \\
2 p_{9}-3 s_{1}^{\prime \prime} \\
2 p_{6}-5 d_{5} \\
3 d_{1}^{\prime \prime}-6 \mathrm{~W}^{*} \\
3 d_{1}^{\prime \prime}-6 \mathrm{Y}^{\circ}\end{array}$ & $\begin{array}{l}.92 \\
.86 \\
.825 \\
.86 \\
.30\end{array}$ \\
\hline $\begin{array}{c}30 \\
3 \\
20 \\
30 \\
1 h\end{array}$ & $\begin{array}{l}7,019.02 \\
7,034.80 \\
7,035.53 \\
7,047.37 \\
7,049.07\end{array}$ & $\begin{array}{l}14,243.08 \\
14,211.13 \\
14,209.66 \\
14,185.79 \\
14,182.36\end{array}$ & $\begin{array}{l}2 p_{5}-5 d_{4} \\
2 p_{6}-4 s_{4} \\
2 p_{6}-4 s_{5} \\
3 d_{5}-5 p_{7}\end{array}$ & $\begin{array}{l}.073 \\
.645 \\
.788 \\
.21\end{array}$ & $\begin{array}{r}1 \\
100 \\
10 \\
15 \\
100\end{array}$ & \begin{tabular}{|l|}
$7,790.53$ \\
$7,802.651$ \\
$7,832.98$ \\
$7,841.23$ \\
$7,881.320$
\end{tabular} & $\begin{array}{l}12,832.57 \\
12,812.636 \\
12,763.02 \\
12,749.60 \\
12,684.745\end{array}$ & $\begin{array}{c}s c_{1}^{\prime \prime}-6 U^{\prime} \\
2 p_{9}-3 s_{4} \\
2 p_{10}-4 d_{2} \\
2 p_{8}-3 s_{1}^{\prime \prime} \\
2 p_{0}-3 s_{s}\end{array}$ & $\begin{array}{l}.46 \\
.636 \\
.00 \\
.60 \\
.742\end{array}$ \\
\hline $\begin{array}{c}1 h \\
3 \\
1 \\
500 \\
15 \\
10\end{array}$ & $\begin{array}{l}7,049.36 \\
7,051.06 \\
7,078.46 \\
7,119.598 \\
7,136.57 \\
7,172.70\end{array}$ & \begin{tabular}{l|}
$14,181.78$ \\
$14,178.36$ \\
$14,123.48$ \\
$14,041.872$ \\
$14,008.48$ \\
$13,937.92$
\end{tabular} & $\begin{array}{c}3 d_{1}{ }^{\prime \prime}-\delta \mathrm{V} \\
2 p_{10}-3 s^{\prime \prime \prime \prime \prime} \\
3 d_{5}-5 p_{9} \\
2 p_{5}-5 d_{4}^{\prime} \\
2 p_{9}-5 d_{5} \\
3 d_{4}^{\prime}-5 p_{8}\end{array}$ & \begin{tabular}{l|}
.75 \\
.34 \\
.50 \\
.872 \\
.491 \\
.88
\end{tabular} & $\begin{array}{r}300 \\
40 \\
4 \\
500 \\
8\end{array}$ & $\begin{array}{l}7,887.395 \\
7,937.41 \\
7,954.22 \\
7,967.341 \\
7,976.03\end{array}$ & $\begin{array}{l}12,674.975 \\
12,595.11 \\
12,568.49 \\
12,547.792 \\
12,534.12\end{array}$ & $\begin{array}{l}1 s_{2}-2 p_{1} \\
2 p_{5}-5 d_{2} \\
3 d_{5}-4 p_{5} \\
1 s_{3}-3 p_{i} \\
2 p_{6}-3 s_{1}^{\prime \prime \prime}\end{array}$ & $\begin{array}{l}.978 \\
.081 \\
.50 \\
.789 \\
.100\end{array}$ \\
\hline $\begin{array}{r}15 \\
5 \\
1 \\
3 \\
20\end{array}$ & $\begin{array}{l}7,200.79 \\
7,209.14 \\
7,220.24 \\
7,238.20 \\
7,244.94\end{array}$ & $\begin{array}{l}13,883.54 \\
13,867.46 \\
13,846.14 \\
13,811.79 \\
13,798.94\end{array}$ & $\begin{array}{c}2 p_{10}-3 s_{1}{ }^{\prime \prime} \\
3 d_{3}-5 p_{6} \\
3 d_{3}-5 p_{7} \\
3 d_{3}-5 p_{8} \\
3 d_{4}-6 W^{-}\end{array}$ & \begin{tabular}{r|}
.51 \\
.47 \\
.09 \\
.76 \\
9.00
\end{tabular} & $\begin{array}{r}10 \\
100 \\
10 \\
15 \\
200\end{array}$ & $\begin{array}{l}8,003.26 \\
8,029.67 \\
8,040.56 \\
8,042.18 \\
8,057.258\end{array}$ & $\begin{array}{l}12,491.48 \\
12,450.39 \\
12,433.53 \\
12,431.02 \\
12,407.762\end{array}$ & $\begin{array}{l}2 p_{7-}-3 s_{1}{ }_{1 \prime \prime \prime} \\
3 d_{4}-5 W \\
3 d_{4}-5 \bigvee \\
3 d_{4}-5 Z \\
3 d_{4}-5 Z\end{array}$ & $\begin{array}{l}.45 \\
.41 \\
.51 \\
.02 \\
.75\end{array}$ \\
\hline 2 & $7,249.92$ & $13,789.46$ & & .44 & $\begin{array}{r}150 \\
2\end{array}$ & & $\begin{array}{l}12,401.480 \\
12,395.94\end{array}$ & $\begin{array}{l}2 p_{5}-3 s_{5} \\
3 d_{4}-5 \mathrm{I}\end{array}$ & $\begin{array}{l}.483 \\
.79\end{array}$ \\
\hline $5 h$ & $7,250.87$ & $13,787.66$ & & .38 & $\begin{array}{l}1 \\
3\end{array}$ & & & & $\begin{array}{l}.011 \\
43\end{array}$ \\
\hline $\begin{array}{l}60 \\
20\end{array}$ & $\begin{array}{l}7,257.94 \\
7,262.54\end{array}$ & $\begin{array}{l}13,774.22 \\
13,765.50\end{array}$ & $\begin{array}{l}3 d_{4}-6 Z \\
2 p_{i}-5 d_{3}\end{array}$ & \begin{tabular}{l|}
.23 \\
.505
\end{tabular} & 100 & $8,101.98$ & 12 , & & 26 \\
\hline $\begin{array}{c}25 \\
40 \\
60 \\
5 h \\
1 h\end{array}$ & $\begin{array}{l}7,266.49 \\
7,283.961 \\
7,285.301 \\
7,307.37 \\
7,313.01\end{array}$ & \begin{tabular}{|l|}
$13,758.02$ \\
$13,725.018$ \\
$13,722.494$ \\
$13,681.05$ \\
$13,670.50$
\end{tabular} & $\begin{array}{l}2 p_{i}-5 d_{2} \\
1 s_{2}-4 V^{\prime} \\
2 p_{\overline{7}}-5 d_{1}^{\prime \prime} \\
3 d_{1}^{\prime}-8 \mathbb{T}^{\prime} \\
3 d_{1}^{\prime}-8 Z\end{array}$ & $\begin{array}{l}.017 \\
.023 \\
.494 \\
.01 \\
.56\end{array}$ & $\begin{array}{r}15 \\
15 \\
2 \\
2\end{array}$ & $\begin{array}{l}8,107.91 \\
8,109.46 \\
8,118.29 \\
8,123.29 \\
8,165.37\end{array}$ & $\begin{array}{l}12,330.25 \\
12,327.89 \\
12,314.48 \\
12,306.90 \\
12,243.48\end{array}$ & $\begin{array}{l}3 d_{1}^{\prime}-6 \mathrm{~V} \\
3 d_{1}^{\prime}-6 \mathrm{U} \\
3 d_{1}^{\prime}-6 \mathrm{Z} \\
3 d_{1}^{\prime}-6 \mathrm{Y}^{-}\end{array}$ & $\begin{array}{l}9.71 \\
.86 \\
.49 \\
.93\end{array}$ \\
\hline $\begin{array}{r}70 \\
20 \\
15 \\
80 \\
2\end{array}$ & $\begin{array}{l}7,316.272 \\
7,316.87 \\
7,319.94 \\
7,312.452 \\
7,323.05\end{array}$ & $\begin{array}{l}13,664.405 \\
13,663.28 \\
13,657.56 \\
13,654.737 \\
13,651.76\end{array}$ & $\begin{array}{c}1 s_{2}-4 \mathrm{Y} \\
2 p_{10}-3 s_{4} \\
3 d_{1}^{\prime \prime}-7 \mathrm{~W} \\
1 \varepsilon_{2}-4 \mathrm{X} \\
3 d_{1}^{\prime \prime}-7 \mathrm{~V}\end{array}$ & $\begin{array}{l}.408 \\
.290 \\
.52 \\
.740 \\
.59\end{array}$ & $\begin{array}{r}100 \\
1- \\
2 \\
700 \\
10,000\end{array}$ & $\begin{array}{l}8,171.02 \\
8,182.93 \\
8,196.73 \\
8,206.341 \\
8,231.6348\end{array}$ & $\begin{array}{l}12,235.01 \\
12,217.21 \\
12,196.63 \\
12,182.354 \\
12,144.921\end{array}$ & $\begin{array}{l}3 d_{5}-4 p_{p} \\
2 p_{6}-3 s_{1}^{\prime \prime \prime} \\
3 d_{2}-8 V^{\prime \prime} \\
2 p-3 s_{1}^{\prime \prime} \\
1 s_{5}-2 p_{4} \\
1 s_{5}-2 p_{6}\end{array}$ & $\begin{array}{l}4.74 \\
.02 \\
.40 \\
.62 \\
.355 \\
.923\end{array}$ \\
\hline $\begin{array}{r}50 \\
40 \\
100 \\
150 \\
30\end{array}$ & $\begin{array}{l}7,336.480 \\
7,355.58 \\
7,386.002 \\
7,393.793 \\
7,400.41\end{array}$ & $\begin{array}{l}13,626.76 \pi \\
13,591.38 \\
13,535.397 \\
13,521.140 \\
13,509.04\end{array}$ & $\begin{array}{c}2 p_{g}-3 s_{1}{ }^{\prime \prime \prime} \\
3 d_{6}-5 \mathrm{X} \\
2 p_{10}-3 s_{5} \\
2 p_{6}-5 d_{1}^{\prime} \\
2 p_{6}-5 d_{3}\end{array}$ & \begin{tabular}{l|}
.767 \\
.401 \\
.396 \\
.137 \\
.077
\end{tabular} & $\begin{array}{r}500 \\
7,000 \\
15 \\
2 \\
20\end{array}$ & $\begin{array}{l}8,266.519 \\
8,280.1163 \\
8,297.71 \\
8,323.90 \\
8,324.58\end{array}$ & \begin{tabular}{l|}
$12,093.671$ \\
$12,073.811$ \\
$12,048.21$ \\
$12,010.31$ \\
$12,009.32$
\end{tabular} & & $\begin{array}{l}.673 \\
.811 \\
.16 \\
.31 \\
.301\end{array}$ \\
\hline $\begin{array}{r}12 \\
3 \\
20 \\
20 \\
25\end{array}$ & $\begin{array}{l}7,404.51 \\
7,405.77 \\
7,424.05 \\
7,441.94 \\
7,451.00\end{array}$ & $\begin{array}{l}13,501.57 \\
13,499.26 \\
13,466.03 \\
13,433.66 \\
13,417.33\end{array}$ & $\begin{array}{l}2 p_{6}-5 d_{2} \\
2 p_{5}-2 s_{2} \\
2 p_{6}-5 d_{1}^{\prime \prime} \\
2 p_{6}-5 d_{4} \\
3 d_{5}-5 \mathrm{~V}\end{array}$ & $\begin{array}{l}.585 \\
.28 \\
.062 \\
.665 \\
.33\end{array}$ & $\begin{array}{r}2,000 \\
60 \\
40 \\
3 \\
5\end{array}$ & $\begin{array}{l}8,346.823 \\
8,347.45 \\
8,349.05 \\
8,371.38 \\
8,372.79\end{array}$ & $\begin{array}{l}11,977.318 \\
11,976.42 \\
11,974.12 \\
11,942.18 \\
11,940.17\end{array}$ & $\begin{array}{c}1 s_{2}-2 p_{3} \\
2 p_{i}-3 s_{4} \\
2 p_{10}-4 d_{1}{ }^{\prime \prime} \\
3 d_{3}-4 p_{8} \\
2 p_{6}-3 s_{1}^{\prime \prime}\end{array}$ & $\begin{array}{l}.320 \\
.401 \\
.10 \\
.19 \\
.19\end{array}$ \\
\hline $\begin{array}{r}40 \\
25 \\
20 \\
20 \\
8\end{array}$ & $\begin{array}{l}7,472.01 \\
7,474.01 \\
7,492.23 \\
7,501.13 \\
7,514.54\end{array}$ & $\begin{array}{l}13,379.60 \\
13,376.02 \\
13,343.49 \\
13,327.66 \\
13,303.88\end{array}$ & $\begin{array}{l}3 d_{5}-5 \mathrm{Y} \\
3 d_{5}-5 \mathrm{X} \\
2 p_{8}-3 s_{1}^{\prime \prime \prime} \\
2 p_{8}-3 s_{1}^{\prime \prime \prime} \\
2 p_{i}-5 d_{6}\end{array}$ & \begin{tabular}{l||}
.61 \\
.04 \\
.508 \\
.69 \\
.911
\end{tabular} & $\begin{array}{r}20 \\
5 \\
2,000 \\
10 \\
1 h\end{array}$ & $\begin{array}{l}8,392.37 \\
8,402.03 \\
8,409.190 \\
8,437.55 \\
8,450.37\end{array}$ & \begin{tabular}{|l|}
$11,912.32$ \\
$11,898.62$ \\
$11,888.489$ \\
$11,848.53$ \\
$11,830.55$
\end{tabular} & $\begin{array}{l}2 p_{s}-4 d_{2} \\
3 d_{3}-4 p_{s} \\
1 p_{5}-2 p_{i} \\
2 p_{i}-3 s_{5} \\
3 d_{3}-4 p_{10}\end{array}$ & $\begin{array}{l}.35 \\
.60 \\
.491 \\
.507 \\
.53\end{array}$ \\
\hline $\begin{array}{r}3 \\
40 \\
6 \\
10 \\
200\end{array}$ & $\begin{array}{l}7,514.96 \\
7,559.79 \\
7,570.93 \\
7,584.29 \\
7,584.680\end{array}$ & $\begin{array}{l}13,303.13 \\
13,224.25 \\
13,204.79 \\
13,181.53 \\
13,180.849\end{array}$ & $\begin{array}{l}2 p_{5}-4 s_{4} \\
3 d_{4}^{\prime}-5 W \\
3 d_{4}^{\prime}-5 \mathrm{U} \\
3 d_{4}^{\prime}-5 Z \\
3 d_{i^{\prime}}^{\prime}-5 \mathrm{~T}\end{array}$ & $\begin{array}{l}.141 \\
.23 \\
.84 \\
.57 \\
.85\end{array}$ & $\begin{array}{c}1 \\
30 \\
30 \\
2 \\
1-\end{array}$ & $\begin{array}{l}8,501.02 \\
8,522.55 \\
8,530.10 \\
8,553.97 \\
8,564.7\end{array}$ & $\begin{array}{l}11,760.07 \\
11,730.35 \\
11,719.98 \\
11,687.27 \\
11,672.63\end{array}$ & $\begin{array}{l}1 s_{3}-3 p_{10} \\
2 p_{6}-3 s_{4} \\
3 d_{2}-7 V \\
3 d_{2}-7 Y\end{array}$ & $\begin{array}{l}.360 \\
.969 \\
.24 \\
.60\end{array}$ \\
\hline $\begin{array}{c}6 \\
1 \\
10 \\
2 h \\
5\end{array}$ & $\begin{array}{l}7,589.61 \\
7,594.36 \\
7,600.77 \\
7,604.97 \\
7,608.46\end{array}$ & $\begin{array}{l}13,172.28 \\
13,164.04 \\
13,152.95 \\
13,145.68 \\
13,139.65\end{array}$ & $\begin{array}{c}2 p_{p}-5 d_{5} \\
3 d_{4}-5 p_{8} \\
3 d_{1}^{\prime}-7 \mathrm{~W} \\
3 d_{1}^{\prime}-7 \mathrm{U} \\
3 d_{4}-5 p_{8}\end{array}$ & $\begin{array}{l}.257 \\
.06 \\
.92 \\
.80 \\
.68\end{array}$ & $\begin{array}{r}200 \\
80 \\
250 \\
100 \\
200\end{array}$ & $\begin{array}{l}8,576.01 \\
8,624.24 \\
8,648.54 \\
8,692.20 \\
8,696.86\end{array}$ & $\begin{array}{l}11,657.24 \\
11,592.04 \\
11,559.47 \\
11,501.41 \\
11,495.25\end{array}$ & $\begin{array}{c}1 s_{2}-3 p_{5} \\
2 p_{6}-3 s_{5} \\
1 \varepsilon_{2}-3 p_{7} \\
11_{2}-3 p_{6} \\
3 d_{1}^{\prime \prime}-5 \mathrm{~W}\end{array}$ & $\begin{array}{l}.221 \\
.075 \\
.521 \\
.460 \\
.27\end{array}$ \\
\hline $\begin{array}{r}3 \\
500 \\
100 \\
10 \\
30\end{array}$ & $\begin{array}{l}7,609.82 \\
7,642.025 \\
7,642.30 \\
7,643.91 \\
7,664.02 \\
7,664.56\end{array}$ & $\begin{array}{l}13,137.30 \\
13,081.940 \\
13,081.47 \\
13,078.71 \\
13,044.40 \\
13,043.48\end{array}$ & $\begin{array}{l}3 d_{1}^{\prime}-7 Z \\
13 z_{3}-2 p_{2} \\
3 d_{3}-5 \mathrm{~V} \\
3 d_{3}-5 \mathrm{U} \\
2 p_{5}-3 s_{1}^{\prime \prime \prime \prime} \\
3 d_{3}-5 \mathrm{Y}^{\prime}\end{array}$ & $\begin{array}{l}.31 \\
.941 \\
.21 \\
.72 \\
.43 \\
.49\end{array}$ & $\begin{array}{r}40 \\
2 \\
300 \\
100 \\
5,000\end{array}$ & $\begin{array}{l}8,71.54 \\
8,739.39 \\
8,758.20 \\
8,819.412\end{array}$ & \begin{tabular}{|l|}
$11,478.38$ \\
$11,475.88$ \\
$11,439.31$ \\
$11,414.74$ \\
$11,335.515$
\end{tabular} & $\begin{array}{c}3 d_{1}^{\prime \prime}-5 \mathrm{~V}^{\top} \\
3 d_{1}^{\prime \prime}-5 \mathrm{U} \\
2 p_{10}-4 d_{3} \\
2 p_{9}-4 d_{1}^{\prime} \\
1 s_{5}-2 p_{8}\end{array}$ & $\begin{array}{l}.37 \\
.88 \\
.31 \\
.75 \\
.515\end{array}$ \\
\hline
\end{tabular}


TABLE 1.-List of Xe I lines-Continued

\begin{tabular}{|c|c|c|c|c|c|c|c|c|c|}
\hline $\begin{array}{l}\text { Inten- } \\
\text { sity }\end{array}$ & $\begin{array}{l}\text { Wave } \\
\text { length }\end{array}$ & $\begin{array}{c}\text { Wave } \\
\text { number } \\
\text { observed }\end{array}$ & $\underset{\text { tion }}{\text { Combina- }}$ & $\begin{array}{l}\text { Wave } \\
\text { num- } \\
\text { ber } \\
\text { calcu- } \\
\text { lated }\end{array}$ & $\begin{array}{l}\text { Inten- } \\
\text { sity }\end{array}$ & $\begin{array}{l}\text { Wave } \\
\text { length }\end{array}$ & $\begin{array}{c}\text { Wave } \\
\text { number } \\
\text { observed }\end{array}$ & $\underset{\text { tion }}{\text { Combina- }}$ & $\begin{array}{l}\text { Wave } \\
\text { num- } \\
\text { ber } \\
\text { calcu- } \\
\text { lated }\end{array}$ \\
\hline $\begin{array}{r}1 \\
300 \\
10 \\
200 \\
200\end{array}$ & $\begin{array}{l}8,851.44 \\
8,862.32 \\
8,885.71 \\
8,908.73 \\
8,930.83\end{array}$ & $\begin{array}{l}11,294.50 \\
11,230.63 \\
11,250.94 \\
11,221.87 \\
11,194.10\end{array}$ & $\begin{array}{r}3 d_{4}-4 p_{3} \\
2 p_{10}+d_{5} \\
3 d_{4}-4 p_{9} \\
2 p_{10}+d_{6} \\
1 s_{2}-2 p_{4}\end{array}$ & $\begin{array}{r}.49 \\
.63 \\
.90 \\
.87 \\
4.087\end{array}$ & $\begin{array}{r}3 \\
1 \\
1 \\
1 \\
150\end{array}$ & $\begin{array}{l}9,605.80 \\
9,614.43 \\
9,616.95 \\
9,668.94 \\
9,685.32\end{array}$ & $\begin{array}{l}10,407.53 \\
10,398.18 \\
10,395.46 \\
10,339.56 \\
10,322.08\end{array}$ & $3 d_{1}^{\prime \prime}-4 p_{7}$ & .08 \\
\hline $\begin{array}{r}1,000 \\
50 \\
100 \\
200 \\
30\end{array}$ & $\begin{array}{l}8,952.254 \\
8,952.78 \\
8,981.05 \\
8,987.57 \\
9,025.95\end{array}$ & $\begin{array}{l}11,167.309 \\
11,166.65 \\
11,131.50 \\
11,123.43 \\
11,076.09\end{array}$ & $\begin{array}{l}1 s_{4}-2 p_{8} \\
1 s_{2}-3 p_{9} \\
2 p_{3}-4 d_{1}^{\prime} \\
2 p_{9}-4 d_{1}^{\prime \prime} \\
2 p_{7}-4 d_{3}\end{array}$ & $\begin{array}{l}.307 \\
.641 \\
.49 \\
.45 \\
.11\end{array}$ & $\begin{array}{r}20 \\
2 \\
100 \\
2,000 \\
3,000\end{array}$ & $\begin{array}{l}9,700.99 \\
9,710.03 \\
9,718.16 \\
9,799.699 \\
9,923.192\end{array}$ & $\begin{array}{l}10,305.40 \\
10,295.81 \\
10,287.20 \\
10,201.600 \\
10,074.643\end{array}$ & $\begin{array}{c}2 p_{8}-4 d_{3} \\
3 d_{1}^{\prime \prime}-4 p_{9} \\
2 p_{7}-4 d_{1}^{\prime \prime} \\
1 s_{5}-2 p_{10} \\
1 s_{4}-2 p_{9}\end{array}$ & $\begin{array}{l}.40 \\
.76 \\
.21 \\
.602 \\
.640\end{array}$ \\
\hline $\begin{array}{r}50 \\
400 \\
50 \\
4 \\
3\end{array}$ & $\begin{array}{l}9,032.18 \\
9,045.446 \\
9,096.13 \\
9,112.24 \\
9,131.59\end{array}$ & $\begin{array}{l}11,068.49 \\
11,052.256 \\
10,990.67 \\
10,971.24 \\
10,947.99\end{array}$ & $\begin{array}{r}3 d_{6}-4 X \\
1 s_{5}-2 p_{9} \\
3 d_{1}^{\prime}-5 W \\
3 d_{1}^{\prime}-5 U \\
3 d_{1}^{\prime}-5 Z\end{array}$ & $\begin{array}{l}.502 \\
.256 \\
.67 \\
.28 \\
.01\end{array}$ & $\begin{array}{r}10 \\
50 \\
1 \\
5 \\
10\end{array}$ & $\begin{array}{r}9,966.58 \\
10,023.72 \\
10,056.84 \\
10,057.96 \\
10,060.96\end{array}$ & $\begin{array}{r}10,030.78 \\
9,973.60 \\
9,940.76 \\
9,939.66 \\
9,936.69\end{array}$ & $\begin{array}{l}2 p_{6}-4 d_{1}^{\prime \prime} \\
3 d_{4}-4 \mathrm{~W} \\
3 d_{4}-4 \mathrm{~V} \\
3 d_{4}-4 \mathrm{U}\end{array}$ & $\begin{array}{l}.78 \\
.58 \\
.60 \\
.63\end{array}$ \\
\hline $\begin{array}{r}2 \\
20 \\
2 \\
500 \\
100\end{array}$ & $\begin{array}{l}9,141.8 \\
9,152.12 \\
9,158.38 \\
9,162.654 \\
9,167.52\end{array}$ & $\begin{array}{l}10,935.8 \\
10,923.44 \\
10,915.97 \\
10,910.87 \\
10,905.09\end{array}$ & $\begin{array}{l}3 d_{1}^{\prime}-5 Y \\
3 d_{5}-4 Y \\
1 s_{4}-2 p_{7} \\
2 p_{0}-4 d_{4}\end{array}$ & $\begin{array}{c}6.05 \\
.42 \\
\\
.875 \\
.09\end{array}$ & $\begin{array}{r}20 \\
80 \\
1 \\
20 \\
10\end{array}$ & $\begin{array}{l}10,084.79 \\
10,107.34 \\
10,119.8 \\
10,125.47 \\
10,188.36\end{array}$ & $\begin{array}{l}9,913.21 \\
9,891.10 \\
9,878.88 \\
9,873.38 \\
9,812.44\end{array}$ & $\begin{array}{l}2 p_{5}-4 d_{2} \\
3 d_{4}-4 Z \\
3 d_{4}-4 Y \\
3 d_{5}-2 p_{1} \\
2 p_{8}-4 d_{4}\end{array}$ & $\begin{array}{l}.18 \\
.08 \\
.99 \\
.35 \\
.42\end{array}$ \\
\hline $\begin{array}{r}2 \\
30 \\
25 \\
\frac{1}{5}\end{array}$ & $\begin{array}{l}9,197.18 \\
9,203.20 \\
9,211.38 \\
9,216.51 \\
9,222.39\end{array}$ & $\begin{array}{l}10,869.92 \\
10,862.81 \\
10,853.16 \\
10,847.12 \\
10,840.20\end{array}$ & $\begin{array}{l}3 d_{2}-6 \mathrm{Y} \\
3 d_{5}-4 \mathrm{Y} \\
3 d_{5}-4 \mathrm{X} \\
3 d_{2}-6 \mathrm{Y} \\
2 p_{3}-4 d_{1}^{\prime \prime}\end{array}$ & $\begin{array}{l}.96 \\
.81 \\
.14 \\
.18 \\
.19\end{array}$ & $\begin{array}{r}20 \\
1 \\
8 \\
6 \\
10\end{array}$ & $\begin{array}{l}10,251.07 \\
10,420.52 \\
10,484.83 \\
10,507.91 \\
10,515.15\end{array}$ & $\begin{array}{l}9,752.41 \\
9,593.83 \\
9,534.98 \\
9,514.03 \\
9,507.48\end{array}$ & $\begin{array}{l}2 p_{7}-4 d_{3} \\
2 p_{7}-4 d_{5} \\
2 p_{7}-4 d_{6} \\
3 d_{2}-5 \mathrm{~V} \\
3 d_{6}-2 p_{2}\end{array}$ & $\begin{array}{l}.42 \\
.74 \\
.98 \\
.02 \\
.44\end{array}$ \\
\hline $\begin{array}{r}3 \\
30 \\
40 \\
3 \\
10\end{array}$ & $\begin{array}{l}9,245.18 \\
9,301.95 \\
9,306.64 \\
9,334.08 \\
9,37 \pm .02\end{array}$ & $\begin{array}{l}10,513.45 \\
10,747.49 \\
10,742.07 \\
10,710.49 \\
10,664.86\end{array}$ & $\begin{array}{c}2 p_{5}-3 s_{4} \\
3 d_{4}^{\prime}-4 \mathrm{~W} \\
1 s_{2}-3 p_{10} \\
3 d_{4}^{\prime}-4 \mathrm{U} \\
3 d_{4}^{\prime}-4 Z\end{array}$ & $\begin{array}{l}.465 \\
.40 \\
.092 \\
.45 \\
.90\end{array}$ & $\begin{array}{r}40 \\
4 \\
25 \\
15 \\
100\end{array}$ & $\begin{array}{l}10,527.84 \\
10,549.75 \\
10,706.77 \\
10,758.85 \\
10,838.37\end{array}$ & $\begin{array}{l}9,496.03 \\
9,476.30 \\
9,337.33 \\
9,292.13 \\
9,223.95\end{array}$ & $\begin{array}{l}2 p_{6}-4 d_{3} \\
3 d_{2}-5 \mathrm{Y} \\
2 p_{0}-4 d_{5} \\
3 d_{5}-2 p_{2} \\
1 s_{4}-2 p_{10}\end{array}$ & $\begin{array}{l}5.99 \\
.30 \\
.31 \\
.07 \\
.986\end{array}$ \\
\hline $\begin{array}{r}100 \\
60 \\
20 \\
20 \\
30\end{array}$ & $\begin{array}{l}9,374.76 \\
9,412.01 \\
9,441.46 \\
9,442.68 \\
9,445.31\end{array}$ & $\begin{array}{l}10,664.02 \\
10,62.81 \\
10,588.68 \\
10,587.31 \\
10,584.33\end{array}$ & $\begin{array}{c}3 d_{4}-4 T \\
2 p_{3}-4 d_{4} \\
2 p_{9}-4 d_{3} \\
3 d_{3}-4 V \\
3 d_{3}-4 U\end{array}$ & $\begin{array}{l}.02 \\
.83 \\
.66 \\
.30 \\
.33\end{array}$ & $\begin{array}{r}10 \\
10 \\
2 \\
1\end{array}$ & $\begin{array}{l}10,895.39 \\
11,085.39 \\
11,127.29 \\
11,140.90\end{array}$ & $\begin{array}{l}9,175.68 \\
9,018.42 \\
8,984.46 \\
8,973.48\end{array}$ & $\begin{array}{r}3 d_{5}-2 p_{3} \\
3 d_{1}^{\prime \prime}-4 W \\
3 d_{1}^{\prime \prime}-4 V\end{array}$ & $\begin{array}{l}.72 \\
.44 \\
.46\end{array}$ \\
\hline $\begin{array}{r}4 \\
40 \\
10 \\
200 \\
20\end{array}$ & $\begin{array}{l}9,457.76 \\
9,497.07 \\
9,505.78 \\
9,513.379 \\
9,5 \times 5.14\end{array}$ & $\begin{array}{l}10,537.01 \\
10,526.65 \\
10,517.03 \\
10,505.633 \\
10,429.96\end{array}$ & $\begin{array}{l}3 d_{3}-4 \mathrm{Y} \\
3 d_{3}-4 \mathrm{X} \\
2 p_{3}-4 d_{4}^{\prime} \\
2 p_{0}-4 d_{5}\end{array}$ & $\begin{array}{l}.69 \\
.02 \\
.633 \\
.93\end{array}$ & & & & & \\
\hline
\end{tabular}

\section{DISCUSSION OF Xe I TERMS}

The numerical values of previously known terms have been subject to only slight revisions. The new term table incorporates the changes due to more precise data, the extensions of series, new low terms and changes in the assignment of quantum numbers. The values of the $1 s, 2 p$, and $3 p$ terms as determined from interference measurements (RP245) form the basis of the present table. The numerical values of a considerable number of terms can now be completely determined from interference measurements. These are given to three places of decimals. Table 2 gives the final set of terms and effective quantum numbers. In calculating the effective quantum numbers for non-Ritzian terms, the value of the displacement constant $A$ was taken as 10,540 , representing the level separation of the ground doublet $\left({ }^{2} \mathrm{P}^{0}{ }_{1 / 2 / 2}, 3 / 2\right)$ identified ${ }^{9}$ in the $\mathrm{Xe}$ II spectrum. 


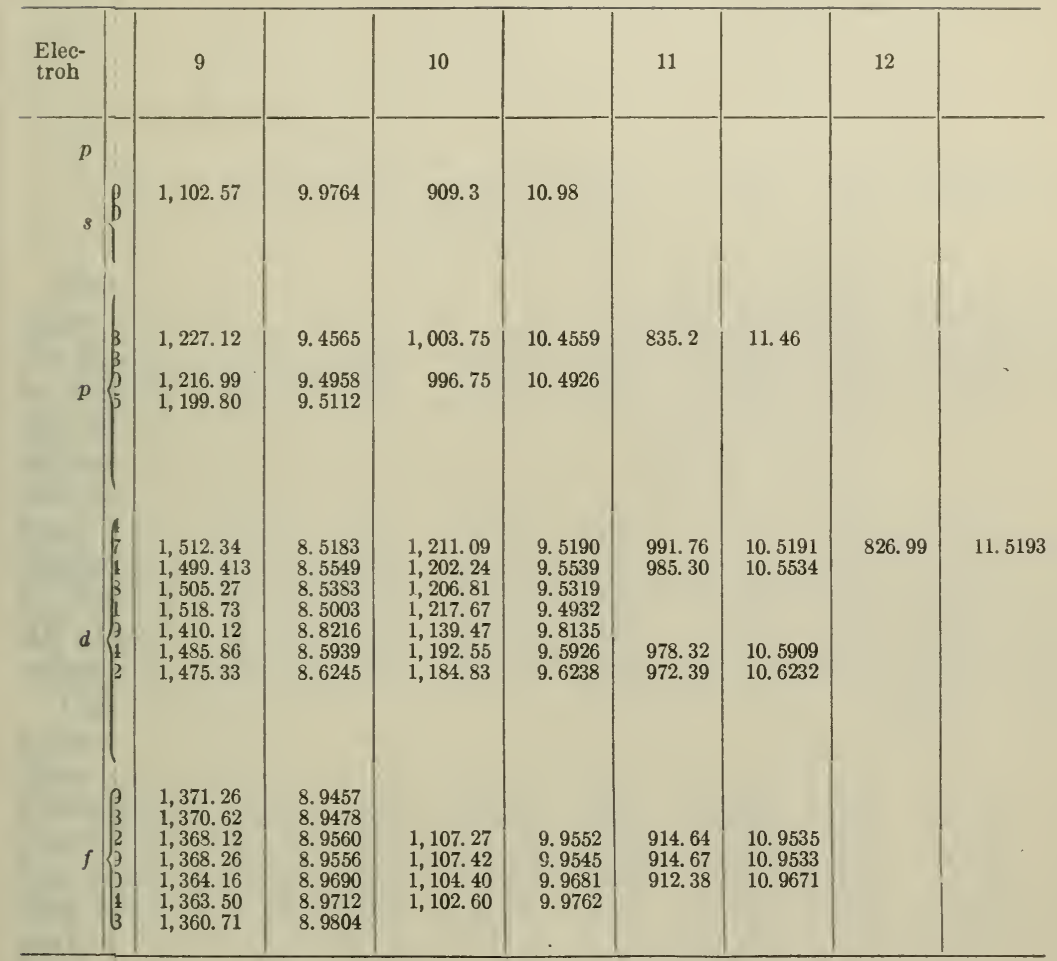

148938-33. (Face p. 146.) 

TABLE 2.-Terms and effective quantum numbers of $X I$

\begin{tabular}{|c|c|c|c|c|c|c|c|c|c|c|c|c|c|c|c|c|c|c|c|c|c|c|c|c|c|c|c|}
\hline $\begin{array}{l}\text { Elec- } \\
\text { troh }\end{array}$ & $j$ & $\begin{array}{c}\text { Pas- } \\
\text { chen } \\
\text { notota- } \\
\text { tion } \\
\end{array}$ & & 1 & & 2 & & 3 & & 4 & & 5 & & 6 & & 7 & & 8 & & 9 & & 10 & & 11 & & 12 & \\
\hline$s$ & $\begin{array}{l}0 \\
2 \\
1 \\
0 \\
1\end{array}$ & $\begin{array}{l}p_{0} \\
s_{3} \\
s_{4} \\
8_{3} \\
s_{2}\end{array}$ & $07,834.8$ & 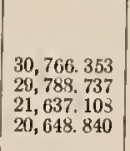 & $\begin{array}{l}1.8886 \\
1.9193 \\
1.8467 \\
1.8758\end{array}$ & $4,215.65$ & 2.7271 & $\begin{array}{l}7,029.355 \\
6,901.461\end{array}$ & $\begin{array}{l}3.9511 \\
3.9876\end{array}$ & $\begin{array}{l}4,435.642 \\
4,411.785\end{array}$ & $\begin{array}{l}\text { 4. } 9739 \\
\text { 4. } 9873\end{array}$ & $\begin{array}{r}3,073.969 \\
3,046.797\end{array}$ & $\begin{array}{l}5.9748 \\
6.0014\end{array}$ & \begin{tabular}{|l}
$2,254.931$ \\
$2,242.92$
\end{tabular} & $\begin{array}{l}\text { 6. } 9760 \\
6.9947\end{array}$ & $\begin{array}{l}1,724.67 \\
1,711.12\end{array}$ & $\begin{array}{l}7.9767 \\
8.0082\end{array}$ & $\begin{array}{l}1,361.76 \\
1,353.27\end{array}$ & $\begin{array}{l}8.9769 \\
9.0050\end{array}$ & $1,102.5 \bar{T}$ & 9.9764 & 009.3 & 10.08 & & & & \\
\hline$p$ & $\begin{array}{l}1 \\
2 \\
3 \\
1 \\
2 \\
0 \\
1 \\
2 \\
1 \\
0\end{array}$ & $\begin{array}{l}p_{10} \\
p_{0} \\
p_{9} \\
p_{p} \\
p_{8} \\
p_{8} \\
p_{4} \\
p_{3} \\
p_{2} \\
p_{1}\end{array}$ & & & & 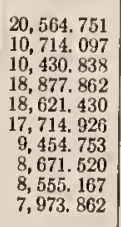 & 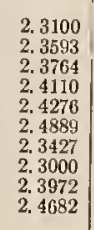 & 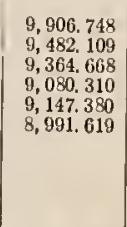 & $\begin{array}{l}3.3282 \\
3.4019 \\
3.4232 \\
3.4764 \\
3.4636 \\
3.4935\end{array}$ & $\begin{array}{l}5,680,591 \\
5,612.51 \\
5,568.980 \\
5,500.812 \\
5,462.985 \\
5,278,741\end{array}$ & $\begin{array}{l}4.3952 \\
4.4218 \\
4.4391 \\
4.4665 \\
4.4819 \\
4.5595\end{array}$ & $\begin{array}{l}3,766.94 \\
3,723.74 \\
33,699.36 \\
3,665.03 \\
33,643.65 \\
3,648.39 \\
3,54.39\end{array}$ & $\begin{array}{l}5.3974 \\
5.4286 \\
5.4466 \\
5.4719 \\
5.4870 \\
5.4611 \\
5.6611\end{array}$ & $\begin{array}{l}2,679.52 \\
2,652.23 \\
2,637.36 \\
2,637.36 \\
2,617.43 \\
2,604.30 \\
2,547.83\end{array}$ & $\begin{array}{l}6.3994 \\
6.4324 \\
66.4505 \\
6.4750 \\
60.4913 \\
6.5028\end{array}$ & $\begin{array}{l}1,975.71 \\
1,963.20 \\
1,954.49 \\
1,918.36\end{array}$ & $\begin{array}{l}7.4528 \\
7.4764 \\
7.4931 \\
7.5633\end{array}$ & $\begin{array}{l}1,534.94 \\
1,527.03 \\
1,521.00 \\
1,496.07\end{array}$ & $\begin{array}{l}8.4553 \\
8.4773 \\
8.4900 \\
8.6645\end{array}$ & $\begin{array}{l}1,227.12 \\
1,216,99 \\
1,199.80\end{array}$ & $\begin{array}{l}9.4565 \\
9.4058 \\
0.5112\end{array}$ & $\begin{array}{r}1,003.75 \\
003.75\end{array}$ & $\begin{array}{l}10.4569 \\
\text { 10. } 1926 \mathrm{f}\end{array}$ & $\$ 35,2$ & 11.16 & & \\
\hline d & $\begin{array}{l}0 \\
4 \\
3 \\
3 \\
1 \\
1 \\
1 \\
2 \\
3 \\
2 \\
2 \\
2 \\
3 \\
1\end{array}$ & 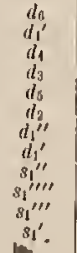 & & & & & & 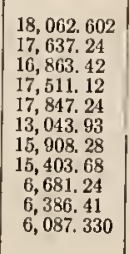 & $\begin{array}{l}2.4648 \\
2.4944 \\
2.5510 \\
2.55035 \\
2.4797 \\
2.8053 \\
2.6264 \\
2.6691 \\
2.5243 \\
2.5462 \\
2.5600\end{array}$ & 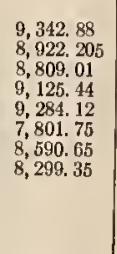 & $\begin{array}{l}3.4272 \\
3.5070 \\
3.5295 \\
3.4678 \\
3.4380 \\
3.7500 \\
3.5741 \\
3.6363\end{array}$ & 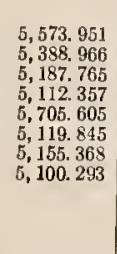 & $\begin{array}{l}4.4370 \\
4.5126 \\
4.5992 \\
4.69330 \\
4.3856 \\
4.6297 \\
4.6137 \\
4.6385\end{array}$ & 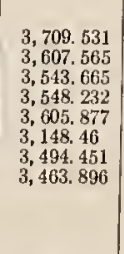 & $\begin{array}{l}5.4390 \\
5.5153 \\
5.5648 \\
55.5612 \\
5.5168 \\
55.9037 \\
5.6039 \\
5.6285\end{array}$ & 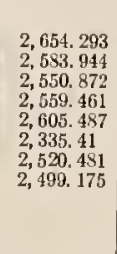 & $\begin{array}{l}6.4298 \\
8.5168 \\
6,5589 \\
6.5479 \\
66.4897 \\
6.8587 \\
6.6084 \\
6.6264\end{array}$ & $\begin{array}{l}2,033.308 \\
1,941.698 \\
1,921.877 \\
1,929.872 \\
1,921.012 \\
1,788.12 \\
1,7002.09 \\
1,887.277\end{array}$ & $\begin{array}{l}7.3 .164 \\
7.5177 \\
77.5564 \\
77.5418 \\
77.5581 \\
77.8339 \\
7.5395 \\
7.6525\end{array}$ & $\begin{array}{l}1,512.34 \\
1,499.413 \\
1,505.27 \\
1,518.73 \\
1,410.12 \\
1,48.56 \\
1,475.33\end{array}$ & $\begin{array}{l}8.518 .3 \\
8.5549 \\
8.5383 \\
8.5003 \\
8.8213 \\
8.5039 \\
8.6245\end{array}$ & 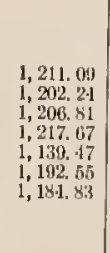 & 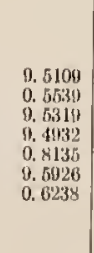 & $\begin{array}{l}151.71 \\
055.31 \\
\\
978.32 \\
072.39\end{array}$ & 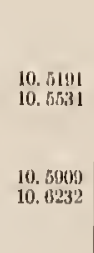 & 826. 90 & 11.6193 \\
\hline$f$ & $\begin{array}{l}1 \\
2 \\
4 \\
5 \\
3 \\
3 \\
3\end{array}$ & $\begin{array}{l}X \\
Y \\
Z \\
T \\
T \\
U \\
Y \\
W\end{array}$ & & & & & & & & $\begin{array}{l}6,994.100 \\
6,994.432 \\
6,972.34 \\
6,973.22 \\
6,992.786 \\
6,923.817 \\
6,989.84\end{array}$ & $\begin{array}{l}3.9610 \\
3.9638 \\
3.9672 \\
3.9670 \\
3.9302 \\
3.9311 \\
3.9909\end{array}$ & 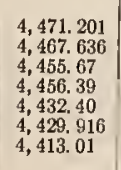 & $\begin{array}{l}4.9511 \\
4.9561 \\
4.9627 \\
4.9623 \\
4.9757 \\
4.9777 \\
4.9866\end{array}$ & $\begin{array}{l}3,098.089 \\
3,099.746 \\
3,099.19 \\
3,099.67 \\
3,075.82 \\
3,073.985 \\
3,064.42\end{array}$ & $\begin{array}{l}5.9507 \\
5.9528 \\
55.9601 \\
55.9596 \\
5.9731 \\
55.9748 \\
5.9841\end{array}$ & $\begin{array}{l}2,272,818 \\
2,271.321 \\
2,266.37 \\
2,260.75 \\
2,257.92 \\
2,256.92 \\
2,250.69\end{array}$ & $\begin{array}{l}6.0484 \\
6.9508 \\
66.9584 \\
6.9587 \\
6.9715 \\
6.9733 \\
6.9325\end{array}$ & $\begin{array}{l}1,737.64 \\
1,736.58 \\
1,733.12 \\
1,733.29 \\
1,723.58 \\
1,726.53 \\
1,722.67\end{array}$ & $\begin{array}{l}7.9469 \\
7.99993 \\
77.9572 \\
77.9569 \\
77.9700 \\
77.9724 \\
7.9813\end{array}$ & $\begin{array}{l}1,371.26 \\
1,370.62 \\
1,368.12 \\
1,368.26 \\
1,364.16 \\
1,363.50 \\
1,360.71\end{array}$ & $\begin{array}{l}8.9457 \\
8.917 \\
8.0560 \\
8.9556 \\
8.9600 \\
8.0712 \\
8.980 .1\end{array}$ & $\begin{array}{l}1,107.27 \\
1,107.42 \\
1,101.40 \\
1,102,60\end{array}$ & $\begin{array}{l}0.9562 \\
0.9515 \\
0.06851 \\
0.0762\end{array}$ & $\begin{array}{l}014.61 \\
9111.07 \\
012,38\end{array}$ & $\begin{array}{l}10.9635 \\
10.0633 \\
10.0671\end{array}$ & & \\
\hline
\end{tabular}




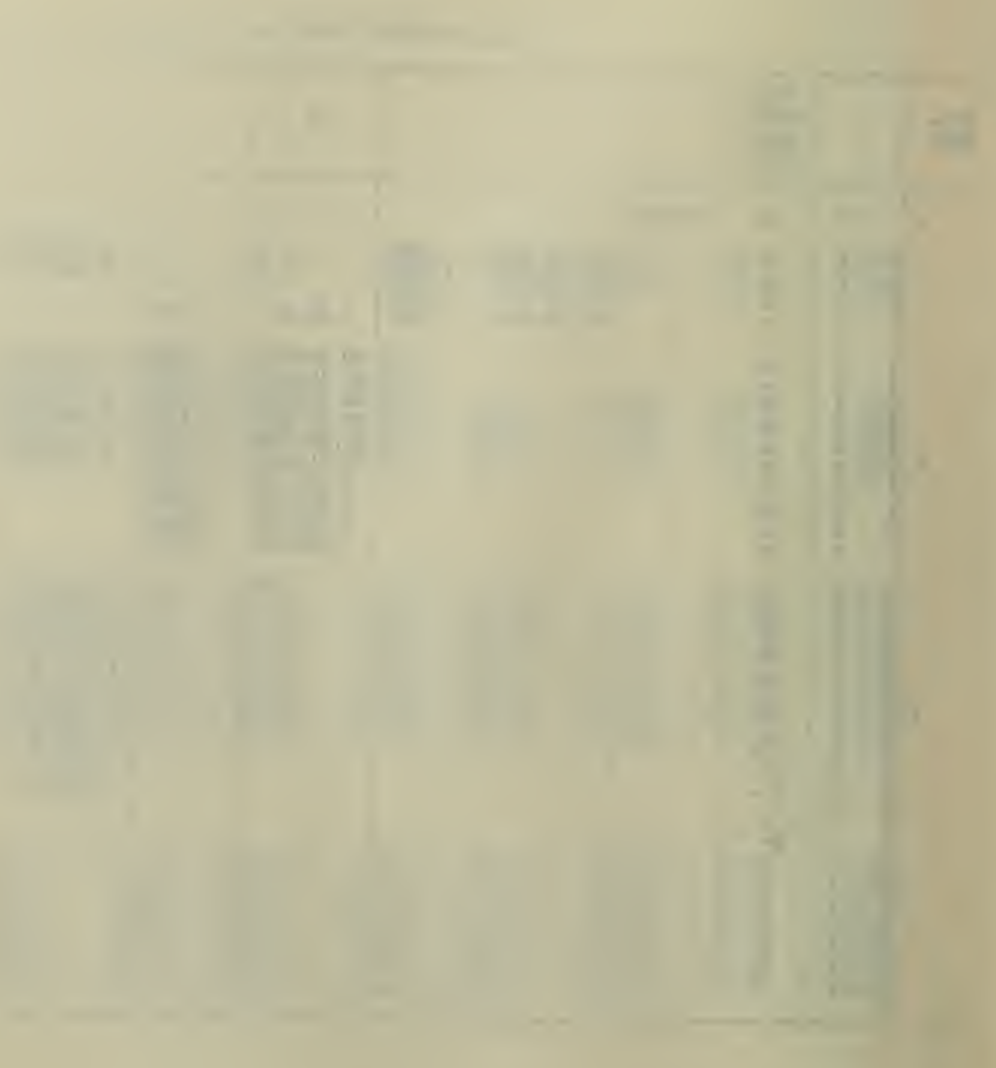


TABLE 3.-Determination of absolute term values of $\mathrm{XeI}$

$$
\begin{aligned}
2 p_{8} & =m d_{4}^{\prime} \quad\left(5_{p}^{3} D_{3}-5 d^{3} F_{4}\right) \\
\nu & =A-\frac{R}{[m+\mu+\alpha(-1-\nu)]^{2}} \\
R_{x \Delta} & =109,736.965 \\
A & =19,430.838 \\
\mu & =+0.5208392314 \\
\alpha & =-0.000001534768614
\end{aligned}
$$

\begin{tabular}{|c|c|c|c|c|c|}
\hline$m$ & Int. & $\lambda_{\text {obs. }}$ & Vobs. & $\nu_{\text {calc }}$ & $\nu_{\text {cale }}-v_{\text {ubu }}$ \\
\hline $\begin{array}{r}2 \\
3 \\
4 \\
5 \\
6 \\
7 \\
5 \\
9 \\
10 \\
11\end{array}$ & $\begin{array}{r}200 \\
500 \\
300 \\
100 \\
70 \\
40 \\
6 h \\
2 h \\
1 h-\end{array}$ & $\begin{array}{l}9,513.32 \\
7,119.598 \\
6,318.062 \\
5,934.170 \\
5,716.255 \\
5,579.276 \\
5,487.026 \\
5,421.756 \\
5,373.74\end{array}$ & $\begin{array}{l}{[1,793.60]} \\
10,508.70 \\
14,041.872 \\
15,823.272 \\
16,846.900 \\
17,489.131 \\
17,918.51 \\
18,219.76 \\
18,439.10 \\
18,603.85\end{array}$ & $\begin{array}{r}1,784.900 \\
10,509.189 \\
14,041.873 \\
15,823.272 \\
16,846.946 \\
17,489.217 \\
17,918.584 \\
18,219.760 \\
18,439.144 \\
18,603.886\end{array}$ & $\begin{array}{c}+8.70 \\
-.48 \\
-.001 \\
-.000 \\
-.046 \\
-.086 \\
=.07 \\
-.000 \\
-.04 \\
-.04\end{array}$ \\
\hline
\end{tabular}

$$
\begin{aligned}
2 p_{s} & =19,430.838 \\
1 s_{s}-2 p_{8} & =11,335.51 j \\
\therefore 1 s_{s} & =30,766.353
\end{aligned}
$$

The absolute value of the term $1 s_{5}$ has been redetermined using the more accurate wave length data now available and a new value of the Rydberg constant for xenon calculated from the value of $R \infty$ given by Birge. ${ }^{10}$ The new value of $1 s_{5}$ differs very slightly from the old, $30,766.353$ as compared with $30,766.98$ previously calculated. This revised term value represents the precision attainable with the data selected for the determination. It is still subject to a variation of from 1 to 2 wave number units, depending on the series chosen for computation. As in the work previously reported, we have estimated $1 s_{5}$ from the limit of the series $2 p_{8}-m d^{\prime}{ }_{4}$ and the combination $1 s_{5}-2 p_{3}$. All other terms are fixed relative to $1 s_{5}$, so that the error in absolute value is the same for all terms.

The sequence $m s_{5}$ remains unchanged except that it has been extended to the tenth member. Attempts to find $2 s_{5}$ from combina tions in the infra-red region have not been successful, and it seems probable that the required combinations are beyond our range of photographic observations. The sequence $m s_{4}$ has also been extended. The term 13,943.93 previously given as $2 s_{4}$ is now interpreted as $3 d_{2}$. The reason for the new assignment will be discussed in connection with the $d$-series. The old terms, $2 s_{3}$ and $2 s_{2}$, originally obtained from the interpretation of the ultra-violet data of Abbink and Dorgelo ${ }^{11}$ are not confirmed by expected combinations in the range of the present observations and are not retained. The term, 4,215.65, is a new term, interpreted as $2 s_{2}$ by Rasmussen, and is confirmed by observed combinations.

No changes have been made in the interpretation of the $p$-series. Higher members have been found in the case of all sequences which form Ritz series. The sequence $m p_{8}$ is the longest; it has been extended to $11 p_{8}$

A considerable number of changes have been introduced in the interpretation of the $d$-series. Several new terms have been found 
and all Ritzian series except $m d_{6}$ have been extended. In the work previously reported, the failure to find all expected combinations, due to the faintness of the sources used and the lack of the superior sensitizers now available for the infra-red region, left the assignment of inner quantum numbers uncertain in many cases. With the more extended and accurate data now available these ambiguities have been eliminated and it is believed that the $j$-values are now determined correctly for all the $d$-terms. In the work of Rasmussen referred to above, new interpretations have been placed on many of the old terms and several additional $d$-terms have been found. The present work entirely confirms Rasmussen's assignment of $j$-values, and we agree also on interpretation of the terms in most cases.

The $m d_{6}$-sequence has been altered only by the substitution of the new term $9,342.88$ for $4 d_{6}$ in place of $9,125.44$, which by virtue of its $j$-value 2 is assigned to $4 d_{3}$.

The $m d_{4}^{\prime}$ sequence is left unchanged. It is the longest as well as the most regular of the $d$-series, and has been extended in the present work to $12 d_{4}^{\prime}$. Attention is again called to Table 3 in which a Ritz formula is applied to the series $2 p_{8}-m d_{4}{ }^{\prime}$. The only large departure from the calculated position of a line is in the case of the photographically inaccessible infra-red line $2 p_{8}-3 d_{4}^{\prime}$, at $1,793.60 \mathrm{~cm}^{-1}$. The position of this line is predicted accurately from the known values of both terms, and differs from the position given by the series formula by $8.70 \mathrm{~cm}^{-1}$.

The first term only of the $m d_{4}$ sequence has been changed. The term $16,863.42$, previously assigned to $3 d^{\prime \prime}$, is now designated $3 d_{4}$; $17,511.12$, formerly called $3 d_{4}$, becomes the first term of the new sequence $m d_{3}$ with inner quantum number 2 . Subsequent terms of $m d_{3}$ are assembled from members of other $d$-series incorrectly interpreted. The second number $4 d_{3}$ is $9,125.44$, formerly $4 d_{6}$, the third $5 d_{3}$ is $5,112.357$, formerly $5 d^{\prime \prime}$, whereas the remaining terms of $m d_{3}$ are taken from the old $m d_{5}$ sequence.

The $m d_{5}$ sequence begins as before with $17,847.24$, as $3 d_{5}$. The term $9,284.12$, formerly $4 d_{2}$, is now assigned to $4 d_{5}$. The term $5,705.605$ was formerly designated as $3 s_{1}^{\prime \prime \prime}$. It is now certain that its $j$-value is 1 . Rasmussen designated it as $5 d_{5}$. We give it the same classification with reservations, since its interpretation as the missing non-Ritzian term $3 s_{1}{ }^{\prime}$ seems equally probable. The present classification introduces a sharp downward inflection in the graph of the effective quantum numbers, which is not paralleled for $\mathrm{Kr}$ I. In any case either $5 d_{5}$ or $3 s_{1}^{\prime}$ has not been found.

With the exception of the first and third members, the $m d_{2}$ sequence is made up of newly found terms. The term 13,943.93 is now designated as $3 d_{2}$ instead of $2 s_{4}$. This assignment is supported by the effective quantum number which fits better into this $d$-series than into the $s_{4}$-series where an unexpected deviation from an otherwise fairly regular series would occur. Furthermore, if $2 s_{4}$ is found, $2 s_{5}$ should also be observed, since the combinations would be displaced to shorter wave lengths than those of $2 s_{4}$. The combination of 13,944 with the ground term giving the line, $83,891.2 \mathrm{~cm}^{-1}$, found in Abbink and Dorgelo's data is permitted with either interpretation. The term $5,119.845$, designated $5 d_{2}$, was formerly $5 d_{5}$. Three new terms, $7,801.75$, 3,148.46, 2,335.41, have been interpreted by Rasmussen as 
$4 d_{2}, 6 d_{2}$, and $T d_{2}$, respectively. We have how extended the series to $10 d_{2}$.

The first three members of the $d_{1}^{\prime \prime}$ sequence have been replaced. The first member $15,908.28$, now $3 d_{1}{ }^{\prime \prime}$, was formerly $3 d_{1}{ }^{\prime}$. The term $8,289.42$, appearing in the original table, is not supported by observed combinations and is obviously spurious. It is replaced by $8,590.65$, designated $4 d_{1}^{\prime \prime}$ instead of $4 d_{5}$ as before. The third term, 5,155.368, has been changed to $5 d_{1}{ }^{\prime \prime}$ from $5 d_{2}$. The original interpretation has been retained for subsequent members of the series.

A new term $15,403.68$ has been interpreted by Rasmussen as $3 d_{1}{ }^{\prime}$. The $m d_{1}^{\prime}$ sequence is otherwise unchanged.

Of the original group of $d$-type non.-Ritzian terms, two are retained; 6,087 is designated $3 s_{1}{ }^{\prime \prime \prime}$ instead of $3 s_{1}{ }^{\prime \prime \prime \prime}$, and 6,386 , now $3 s_{1}{ }^{\prime \prime \prime \prime}$, was formerly $3 s_{1}^{\prime \prime}$. These changes were suggested by Rasmussen to make the notation uniform among the rare gas spectra. A new term 6,681 becomes $3 s_{1}{ }^{\prime \prime}$. Our old $3 s_{1}{ }^{\prime}$ term is not confirmed. The only remaining possibility for $3 s_{1}{ }^{\prime}$ is 5,705 as explained above.

Changes in the $f$-type or hydrogenlike terms have been limited to small corrections and extensions of series, except that one entirely new sequence $m T$ has been found. A discrepancy between observed and calculated wave numbers, too great to be accounted for by errors of observation, was found in the case of the series of lines formerly designated $3 d_{4}^{\prime}-m Z$. 'Examination of the first and second members of the series at 9,374 and 7,584 A with the Rowland grating revealed a companion line in each case in the calculated position of the corresponding member of the $m Z$ series. The stronger series was, therefore, given a separate designation $m T$ and the weaker retained as $m Z$. The separation is $0.9 \mathrm{~cm}^{-1}$ for the first member $0.7 \mathrm{~cm}^{-1}$ for the second, the two series merging in the higher members. Members beyond the second can not be resolved not only because the wave number separation is smaller, but also since the wave length difference diminishes much more rapidly as one goes to shorter wave lengths.

The data here presented, because of greater completeness, accuracy, and the elimination of errors, should be regarded as superseding those given in RP115.

Washington, November 3, 1932. 



\title{
Bureau of Standards Journal of Research
}

\author{
CONTENTS OF RECENT NUMBERS - Continued \\ October, 1932 (Vol. 9, No. 4)
}

RP482. The synthesis, purification, and certain pbysical constants of the normal bydrocarbons from pentane to dodecane, of $n$-amyl bromide and of $n$-nonyl bromide. B. J. Mair. Price 5 cents.

RP483. A study of some ceramic bodies of low absorption maturing at temperatures below $1,000^{\circ}$ C. R. F. Geller and D. N. Evans. Price 5 cents.

RP484. The determination of magnesia in phosphate rock. James I. Hoffman. Price 5 cents.

RP485. Collisions of the first and second kind in the positive column of crsium discharge. F. L. Mobler. Price 5 cents.

RP486. The areas and tensile properties of deformed concrete-reinforcement bars. A. H. Slang, L. R. Sweetman, and C. Gough. Price 5 cents.

RP487. A calorimetric method for determining the intrinsic energy of a gas as a function of the pressure. Edward W. Wasbburn. Price 5 cents.

RP488. The photograpbic emulsion: Variables in sensitization by dyes. Burt H. Carroll and Donald Hubbard. Price 5 cents.

RP489. A method for the separation of rhodium from iridium and the gravimetric determination of these metals. Raleigh Gilchrist. Price 5 cents.

RP490. The isoelectric point of silk. Milton Harris. Price 5 cents.

RP491. Effective applied voltage as an indicator of the radiation emitted by an X-ray tube. Lauriston S. Taylor, George Singer, and C. F. Stoneburner. Price 5 cents.

RP492. Column curves and stress-strain diagrams. William R. Osgood. Price 5 cents.

\section{November, 1932 (Vol. 9, No. 5)}

RP 493. The variation with angle of emission of the radiation from metals bombarded by slow electrons. C. Boeckner.

RP494. Nitrogen content of some standard sample steels. J. G. Thompson and E. H. Hamilton.

RP495. Analytical methods for the determination of levulose in crude products. R. F. Jackson, J. A. Mathews, and W. D. Cbase.

RP496. Determination of alumina and silica in steel by the hydrochloric acid residue method. J. G. Thompson and J. S. Acken.

RP497. An analysis of lantbanum spectra (La I, La II, La III). Henry Norris Russell and William F. Meggers. RP 498. Analysis of weigbted silk. Ralpb T. Mease.

RP 499. The heat of formation of hydrogen chloride and some related thermo-dynamic data. Frederick D. Rossini

RP500. Thermal expansion of lead. Peter Hidnert and W. T. Sweeney.

\section{December, 1932 (Vol. 9, No. 6)}

RP501. Isolation of the three xylenes from an Oklahoma petroleum. Joseph D. White and F. W. Rose, jr.

RP502. Characteristic equations of vacuum and gas-filled tungsten-filament lamps. L. E. Barbrow and J. Franklin Meyer.

RP503. The calorimetric determination of the intrinsic energy of gases as a function of the pressure. Data on oxygen and its mixtures with carbon dioxide to 40 atmospberes, at $28^{\circ} \mathrm{C}$. Frederick D. Rossini and Mikkel Frandsen.

RP504. Shear tests of reinforced brick masonry beams. D. E. Parsons, A. H. Stang, and J. W. McBurney. RP505. The measurement of low-voltage X-ray intensities. Lauriston S. Taylor and C. F. Stoneburner.

RP506. A multi-range potentiometer and its application to the measurement of small temperature differences. H. B. Brooks and A. W. Spinks.

RP507. The density of some soda-lime-silica glasses as a function of the composition. F. W. Glaze, J. C. Young, and A. N. Finn.

RP508. Coiled-filament resistance thermometers. C. H. Meyers.

RP509. Deflection of cosmic rays by a magnetic field. L. F. Curtiss.

RP510. The system: $\mathrm{CaO}-\mathrm{P}_{2} \mathrm{O}_{3}$. Elmer T. Carlson.

RP511. A metal-connected glass electrode. M. R. Thompson.

Research papera, where price is given, are already available, and others will be available in the near future as separates for purchase from the Superintendent of Documents, United States

Government Printing Office, Washington, D. C. 